\title{
Integral Equations with Diagonal and Boundary Singularities of the Kernel
}

\author{
Arvet Pedas and Gennadi Vainikko
}

\begin{abstract}
We study the smoothness and the singularities of the solution to Fredholm and Volterra integral equations of the second kind on a bounded interval. The kernel of the integral operator may have diagonal and boundary singularities, information about them is given through certain estimates. The weighted spaces of smooth functions with boundary singularities containing the solution of the integral equation are described. Examples show that the results cannot be improved.
\end{abstract}

Keywords. Fredholm integral equation, Volterra integral equation, weakly singular integral equation, boundary singularities, smoothness of the solution, compact operators

Mathematics Subject Classification (2000). Primary 45M05, secondary 45B05, 45D05

\section{Introduction, formulation of main results, comments}

1.1. Introduction. It is well understood how a diagonal singularity of the kernel of an integral equation of the second kind generates boundary singularities of the solution (more precisely, of the derivatives of the solution). The case of one dimensional Fredholm integral equations has been analysed in [1], [6]-[10], [14], [18]-[20], [23, 24], the case of Volterra integral equations in [2]-[5], [13] and the case of multidimensional integral equations in $[11,15,17,21,22]$. In the present paper, we examine a more complicated situation for the integral equation

$$
u(x)=\int_{a}^{b} K(x, y) u(y) d y+f(x), \quad a<x<b,
$$

where $K(x, y)$ is a $C^{m}$-smooth kernel on $((a, b) \times(a, b)) \backslash$ diag which, in addition to a diagonal singularity (a singularity as $y \rightarrow x$ ), may have different boundary

A. Pedas and G. Vainikko: Institute of Applied Mathematics, University of Tartu, Estonia; arvet.pedas@ut.ee, gennadi.vainikko@ut.ee 
singularities (singularities as $y \rightarrow a, y \rightarrow b, x \rightarrow a$ or $x \rightarrow b$ ). Here $-\infty<a<$ $b<\infty, \operatorname{diag}=\operatorname{diag}\left(\mathbb{R}^{2}\right)=\left\{(x, y) \in \mathbb{R}^{2}: x=y\right\}$.

To formulate the results of the paper, we first characterise more precisely the possible diagonal and boundary singularities of the kernel and introduce the classes of weighted spaces of $C^{m}$-smooth functions on $(a, b)$ to which a solution of equation (1.1) occurs to belong. Without proofs, a formulation of main results of Sections 1.4, 1.6 and 1.7 of the present paper is given also in [16]. Moreover, [16] contains a formulation of some results about integral equations on a system of intervals not included into the present paper.

1.2. Classes of kernels. We denote $\mathbb{R}=(-\infty, \infty), \mathbb{R}_{+}=[0, \infty), \mathbb{Z}=$ $\{\ldots,-1,0,1,2, \ldots\}, \mathbb{Z}_{+}=\{0,1,2, \ldots\}$. By $c, c^{\prime}, c_{1}$ etc. we denote generic constants that may have different values by different occurrances; we write $c_{K}$ if we want to point out that the constant may depend on the kernel $K$.

For $s \in \mathbb{R}$, denote

$$
\kappa_{s}(r)=\left\{\begin{array}{ll}
1, & s<0 \\
1+|\log r|, & s=0 \\
r^{-s}, & s>0
\end{array} \quad(r>0)\right.
$$

In the sequel $m, k, l \in \mathbb{Z}_{+}$whereas $\lambda, \mu, \nu \in \mathbb{R}$. Introduce the following three classes of kernels:

$\mathcal{W}^{m, \nu}=\mathcal{W}^{m, \nu}((a, b) \times(a, b))$ consists of $m$ times continuously differentiable functions $K$ on $((a, b) \times(a, b)) \backslash \operatorname{diag}$ that satisfy there, for all $k, l, k+l \leq m$, the inequalities

$$
\left|\left(\frac{\partial}{\partial x}\right)^{k}\left(\frac{\partial}{\partial x}+\frac{\partial}{\partial y}\right)^{l} K(x, y)\right| \leq c_{m, K} \kappa_{\nu+k}(|x-y|)
$$

$\mathcal{W}^{m, \nu ; \lambda, \mu}=\mathcal{W}^{m, \nu ; \lambda, \mu}((a, b) \times(a, b))$ consists of $m$ times continuously differentiable functions $K$ on $((a, b) \times(a, b)) \backslash$ diag that satisfy there, for all $k, l$, $k+l \leq m$, the inequalities

$$
\left|\left(\frac{\partial}{\partial x}\right)^{k}\left(\frac{\partial}{\partial x}+\frac{\partial}{\partial y}\right)^{l} K(x, y)\right| \leq c_{m, K} \kappa_{\nu+k}(|x-y|)(y-a)^{-\lambda-l}(b-y)^{-\mu-l}
$$

moreover, in case $\nu<0$, the derivatives $\left(\frac{\partial}{\partial x}\right)^{k} K(x, y)$ with $\nu+k<0, k \leq m$, have continuous extensions onto the square $(a, b) \times(a, b)$ including the diagonal; $\mathcal{W}_{\star}^{m, \nu ; \lambda, \mu}=\mathcal{W}_{\star}^{m, \nu ; \lambda, \mu}((a, b) \times(a, b))$ consists of $K \in \mathcal{W}^{m, \nu ; \lambda, \mu}$ that in addition 
to (1.3) satisfy for all $k, l, k+l \leq m$, the strengthened inequalities

$$
\begin{aligned}
\mid\left(\frac{\partial}{\partial x}\right)^{k} & \left(\frac{\partial}{\partial x}+\frac{\partial}{\partial y}\right)^{l} K(x, y) \mid \\
& \leq c_{m, K} \kappa_{\nu+k}(|x-y|) \begin{cases}\frac{(y-a)^{-\lambda-l}(b-y)^{-\mu-l}}{1+|\log (y-a)|} & \text { if } \lambda+l>0 \\
\frac{(y-a)^{-\lambda-l}(b-y)^{-\mu-l}}{1+|\log (b-y)|} & \text { if } \mu+l>0\end{cases}
\end{aligned}
$$

(both inequalities (1.4) must be fulfilled if $\lambda+l>0$ and $\mu+l>0$ ).

For instance, $K \in \mathcal{W}_{\star}^{m, \nu ; 0,0}$ means that, for $0 \leq k \leq m$,

$$
\left|\left(\frac{\partial}{\partial x}\right)^{k} K(x, y)\right| \leq c \kappa_{\nu+k}(|x-y|)
$$

and, for $l \geq 1, k+l \leq m$,

$$
\left|\left(\frac{\partial}{\partial x}\right)^{k}\left(\frac{\partial}{\partial x}+\frac{\partial}{\partial y}\right)^{l} K(x, y)\right| \leq c \kappa_{\nu+k}(|x-y|) \frac{(y-a)^{-l}(b-y)^{-l}}{1+|\log (y-a)(b-y)|}
$$

and $\left(\frac{\partial}{\partial x}\right)^{k} K(x, y)$ is continuous on $(a, b) \times(a, b)$ if $\nu+k<0, k \leq m$. Clearly,

$$
\begin{array}{ll}
\mathcal{W}^{m, \nu} \subset \mathcal{W}_{\star}^{m, \nu ; 0,0} \subset \mathcal{W}^{m, \nu ; 0,0} & \text { for } \nu \geq 0 \\
\mathcal{W}_{\star}^{m, \nu ; \lambda, \mu} \subset \mathcal{W}^{m, \nu ; \lambda, \mu} \subset \mathcal{W}_{\star}^{m, \nu ; \lambda^{\prime}, \mu^{\prime}} & \text { for } \lambda<\lambda^{\prime}, \mu<\mu^{\prime} \\
\mathcal{W}_{\star}^{m, \nu ; \lambda, \mu}=\mathcal{W}^{m, \nu ; \lambda, \mu} & \text { for } \lambda \leq-m, \mu \leq-m .
\end{array}
$$

For $k=l=0$, condition (1.2) yields

$$
|K(x, y)| \leq c_{m, K} \kappa_{\nu}(|x-y|)=c_{m, K} \begin{cases}1, & \nu<0 \\ 1+|\log | x-y||, & \nu=0 \\ |x-y|^{-\nu}, & \nu>0\end{cases}
$$

thus a kernel $K \in \mathcal{W}^{m, \nu}$ is at most weakly singular for $\nu<1$; for $\nu<0$, the kernel is bounded but its derivatives may have diagonal singularities. Most important examples of weakly singular kernels $K \in \mathcal{W}^{m, \nu}$ are given by

$$
\begin{array}{ll}
K(x, y)=g(x, y)|x-y|^{-\nu} & \text { for } 0<\nu<1 \\
K(x, y)=g(x, y) \log |x-y| & \text { for } \nu=0,
\end{array}
$$

where $g$ is a $C^{m}$-smooth function on $[a, b] \times[a, b]$. For a $K \in \mathcal{W}^{m, \nu}, 0 \leq \nu<1$, the kernel $K(x, y)(y-a)^{-\lambda}(b-y)^{-\mu}$ belongs to $\mathcal{W}^{m, \nu ; \lambda, \mu}$ whereas the kernel $K(x, y) \log (y-a) \log (b-y)$ belongs to $\mathcal{W}_{\star}^{m, \nu ; \lambda^{\prime}, \mu^{\prime}}$ for any $\lambda^{\prime}, \mu^{\prime}>0$. 
Under the conditions $\nu<1, \lambda<\min \{1,1-\nu\}, \mu<\min \{1,1-\nu\}$, a kernel $K \in \mathcal{W}^{m, \nu ; \lambda, \mu}$ is still at most weakly singular in the sense that

$$
\sup _{a<x<b} \int_{a}^{b}|K(x, y)| d y \leq c_{0, K} \sup _{a<x<b} \int_{a}^{b} \kappa_{\nu}(|x-y|)(y-a)^{-\lambda}(b-y)^{-\mu} d y<\infty
$$

(see Section 2), and by $\left(T_{K} u\right)(x)=\int_{a}^{b} K(x, y) u(y) d y, a<x<b$, it is defined an integral operator $T_{K}: L^{\infty}(a, b) \rightarrow L^{\infty}(a, b)$, for $m \geq 1$ even $T_{K}: L^{\infty}(a, b) \rightarrow$ $C[a, b]$. Although we assumed in the definitions of the classes $\mathcal{W}^{m, \nu ; \lambda, \mu}$ and $\mathcal{W}_{\star}^{m, \nu ; \lambda, \mu}$ that $K$ is given only for $(x, y) \in((a, b) \times(a, b)) \backslash$ diag, actually a kernel $K \in \mathcal{W}^{m, \nu ; \lambda, \mu}$ and its derivatives up to the order $m-1$ have continuous extensions to $([a, b] \times(a, b)) \backslash$ diag.

1.3. Weighted spaces of smooth functions. For $s \in \mathbb{R}$, denote

$$
w_{s}(r)=\frac{1}{\kappa_{s}(r)}=\left\{\begin{array}{ll}
1, & s<0 \\
\frac{1}{1+|\log r|}, & s=0 \\
r^{s}, & s>0
\end{array} \quad w_{s}^{\star}(r)=\left\{\begin{array}{ll}
1, & s<0 \\
\frac{r^{s}}{1+|\log r|}, & s \geq 0
\end{array} \quad(r>0)\right.\right.
$$

for $s, t \in \mathbb{R}$, define the following weight functions on $(a, b)$ :

$$
w_{s, t}(x)=w_{s, t}^{(a, b)}(x)=w_{s}(x-a) w_{t}(b-x), \quad w_{s, t}^{\star}(x)=w_{s}^{\star}(x-a) w_{t}^{\star}(b-x) .
$$

Clearly, $w_{s, t}(x) \asymp w_{s}(x-a)$ as $x \rightarrow a, w_{s, t}(x) \asymp w_{t}(b-x)$ as $x \rightarrow b$, i.e., in the vicinities of $a$ and $b$ we have, respectively,

$$
c_{1} w_{s}(x-a) \leq w_{s, t}(x) \leq c_{2} w_{s}(x-a), \quad c_{1} w_{s}(b-x) \leq w_{s, t}(x) \leq c_{2} w_{s}(b-x),
$$

where $0<c_{1}<c_{2}<\infty$. Similar relations hold for $w_{s, t}^{\star}(x)$. For $s, t \in \mathbb{R}$, we introduce the following two Banach spaces:

$C^{m, s, t}=C^{m, s, t}(a, b)$ consists of $m$ times continuously differentiable functions $u$ on $(a, b)$ that have a finite norm

$$
\|u\|_{m, s, t}=\sum_{k=0}^{m} \sup _{a<x<b} w_{k+s-1, k+t-1}(x)\left|u^{(k)}(x)\right| ;
$$

$C_{\star}^{m, s, t}=C_{\star}^{m, s, t}(a, b)$ consists of $m$ times continuously differentiable functions $u$ on $(a, b)$ that have a finite norm

$$
\|u\|_{m, s, t}^{\star}=\sum_{k=0}^{m} \sup _{a<x<b} w_{k+s-1, k+t-1}^{\star}(x)\left|u^{(k)}(x)\right| .
$$

Clearly, $C^{m, s, t}(a, b) \subset C_{\star}^{m, s, t}(a, b) \subset C^{m, s^{\prime}, t^{\prime}}(a, b)$ for $s<s^{\prime}, t<t^{\prime}$. We introduce also the following standard spaces of continuous functions: 
$C[a, b]$ is the Banach space of contiuous functions $u$ on the closed interval $[a, b]$ equipped with the norm $\|u\|_{C[a, b]}=\max _{a \leq x \leq b}|u(x)|$;

$B C(a, b)$ is the Banach space of bounded continuous functions $u$ on the open interval $(a, b)$ equipped with the norm $\|u\|_{B C(a, b)}=\sup _{a<x<b}|u(x)|$;

$U C(a, b)$ is the closed subspace of $B C(a, b)$ that consists of $u$ niformly continuous functions on $(a, b)$, equipped with the same supremum norm .

Clearly, a continuous function $u$ on $(a, b)$ has a continuous extension to $[a, b]$ if and only if $u$ is uniformly continuous on $(a, b)$. This enables to identify the spaces $U C(a, b)$ and $C[a, b]$. Notice that $C^{m, s, t}(a, b) \subset C_{\star}^{m, s, t}(a, b) \subset C[a, b]$ for $m \geq 1, s<1, t<1$ (where we identify $C[a, b]$ with $U C(a, b)$ ). Moreover, it follows by the Arzela Lemma that the imbeddings

$$
C^{m, s, t}(a, b) \subset C[a, b], \quad C_{\star}^{m, s, t}(a, b) \subset C[a, b]
$$

are compact for $m \geq 1, s<1, t<1$.

1.4. Main results. For the sake of a comparison, we first formulate a known result (Theorem 1.1). Namely, the singularities of a solution to equation (1.1) are well understood in the case of kernels $K \in \mathcal{W}^{m, \nu}$, the result reads as follows (see [22]-[24]).

Theorem 1.1. Let $K \in \mathcal{W}^{m, \nu}((a, b) \times(a, b))$ and $f \in C^{m, \nu, \nu}(a, b)$ where $m \geq 1$, $\nu<1$. Then any solution $u \in C[a, b]$ of equation (1.1) belongs to $C^{m, \nu, \nu}(a, b)$.

The main results of this paper concern equation (1.1) with kernels from the classes $\mathcal{W}^{m, \nu ; \lambda, \mu}$ and $\mathcal{W}_{\star}^{m, \nu ; \lambda, \mu}$.

Theorem 1.2. Let $K \in \mathcal{W}^{m, \nu ; \lambda, \mu}((a, b) \times(a, b))$ where

$$
m \geq 1, \quad \nu<1, \quad \lambda<\min \{1,1-\nu\}, \quad \mu<\min \{1,1-\nu\} .
$$

Assume that equation (1.1) has a solution $u \in C[a, b]$. Then the following is true:

(i) if $\nu \notin \mathbb{Z}$ and $f \in C^{m, \nu+\lambda, \nu+\mu}(a, b)$, then $u \in C^{m, \nu+\lambda, \nu+\mu}(a, b)$;

(ii) if $f \in C_{\star}^{m, \nu+\lambda, \nu+\mu}(a, b)$, then $u \in C_{\star}^{m, \nu+\lambda, \nu+\mu}(a, b)$ (for $\nu \in \mathbb{Z}$ as well as for $\nu \notin \mathbb{Z})$.

For $\nu \in \mathbb{Z}$, claim (i) occurs to be wrong. In the following theorem we strengthen the condition on the kernel.

Theorem 1.3. Let $\nu \in \mathbb{Z}, K \in \mathcal{W}_{\star}^{m, \nu ; \lambda, \mu}((a, b) \times(a, b)), f \in C^{m, \nu+\lambda, \nu+\mu}(a, b)$ with the parameters satisfying (1.8), and let $u \in C[a, b]$ be a solution of equation (1.1). Then $u \in C^{m, \nu+\lambda, \nu+\mu}(a, b)$. 
Remark 1.4. Assuming $f \in C^{m}[a, b]$ (or even $f \in C^{\infty}[a, b]$ ), the solution of (1.1) still does have the characteristic singularities of functions from the classes $C^{m, \nu+\lambda, \nu+\mu}(a, b)$ or $C_{\star}^{m, \nu+\lambda, \nu+\mu}(a, b)$, in general, hence the claims of Theorems 1.1-1.3 cannot be strengthened.

Comparing Theorems 1.2-1.3 with Theorem 1.1, we observe that the boundary singularity factors $(y-a)^{-\lambda-l}(b-y)^{-\mu-l}$ in estimates (1.3) shift the solution from $C^{m, \nu, \nu}(a, b)$ into $C^{m, \nu+\lambda, \nu+\mu}(a, b)$ or into $C_{\star}^{m, \nu+\lambda, \nu+\mu}(a, b)$. The singularities of the solution are stronger for greater $\lambda$ and $\mu$. On the other hand, for negative $\lambda$ and $\mu$ the solution has milder singularities than the functions from $C^{m, \nu, \nu}(a, b)$ have. For instance, if the conditions of Theorem 1.2 are fulfilled with $\lambda, \mu<-m-\nu+1$, then all derivatives up to the order $m$ of the solution are bounded in $(a, b)$.

Remark 1.5. We have not assumed the uniqueness of the solution $u$ in Theorems 1.1-1.3. With $f=0$, these theorems can be applied to characterise the singularities of eigenfunctions of the operator $T_{K}$ corresponding to nonzero eigenvalues. Recurrently, Theorem 1.1-1.3 are applicable also to generalised eigenfunctions. Thus we obtain, e.g., the following result from Theorem 1.2: if $K \in \mathcal{W}^{m, \nu ; \lambda, \mu}((a, b) \times(a, b)), m \geq 1, \nu<1, \lambda<\min \{1,1-\nu\}, \mu<\min \{1,1-\nu\}$, then the generalised eigenspace $\left\{u \in C[a, b]:\left(z_{0} I-T_{K}\right)^{N} u=0\right\}$ of the integral operator $T_{K}$ corresponding to a nonzero eigenvalue $z_{0}$ belongs to $C^{m, \nu+\lambda, \nu+\mu}(a, b)$ in case $\nu \notin \mathbb{Z}$ and to $C_{\star}^{m, \nu+\lambda, \nu+\mu}(a, b)$ in case $\nu \in \mathbb{Z}$.

1.5. Proof ideas for the main results. For the proof of Theorems 1.2 and 1.3, we will use the technique of compact operators, see Lemmas $1.6-1.9$ below. Note that for $0 \leq \nu<1$, Theorem 1.1 is a consequence of Theorems 1.2 (i) and 1.3 with $\lambda=0, \mu=0$, so we obtain a new proof of Theorem 1.1 in this case.

Lemma 1.6. Let $E$ and $F$ be Banach spaces such that $E \subset F$ densely and continuously, i.e., $E$ is dense in $F$ and $\|u\|_{F} \leq c\|u\|_{E}$ for every $u \in E$. Let $T$ be a linear operator in $F$ that maps $E$ into $E$ and, moreover, let $T: E \rightarrow E$ and $T: F \rightarrow F$ be compact. Assume that the equation $u=T u+f$ with a given $f \in E$ has a solution $u \in F$. Then $u \in E$.

This Lemma follows from the Fredholm theory for compact operators; see [24] for a detailed proof. The claim of the Lemma is clear in the case where the homogenous eqation $u=T u$ has only the trivial solution $u=0$. But we avoid this assumption in order to have a possibility to tackle the smoothness properties of eigenfunctions of the integral operator $T_{K}$, see Remark 1.5.

For the proof of Theorems 1.2 and 1.3 we use Lemma 1.6 with $F=C[a, b]$ and either $E=C^{m, \nu+\lambda, \nu+\mu}(a, b)$ or $E=C_{\star}^{m, \nu+\lambda, \nu+\mu}(a, b)$. Due to (1.7), (1.8), the corresponding imbeddings $E \subset F$ are continuous, even compact; these imbeddings are also dense since $C^{m, \nu+\lambda, \nu+\mu}(a, b)$ contains $C^{m}[a, b]$. 
Lemma 1.7. Let $K \in \mathcal{W}^{0, \nu ; \lambda, \mu}([a, b] \times(a, b))$ with $\nu<1$ and $\lambda, \mu<\min \{1,1-\nu\}$, i.e., $K$ is continuous on $([a, b] \times(a, b)) \backslash$ diag and

$$
|K(x, y)| \leq c_{K} \kappa_{\nu}(|x-y|)(y-a)^{-\lambda}(b-y)^{-\mu}, \quad(x, y) \in([a, b] \times(a, b)) \backslash \operatorname{diag},
$$

with parameters $\nu, \lambda, \mu$ that satisfy

$$
\nu<1, \quad \lambda<1, \quad \lambda+\nu<1, \quad \mu<1, \quad \mu+\nu<1 .
$$

Then $T_{K}: L^{\infty}(a, b) \rightarrow C[a, b]$ is compact, i.e., $T_{K}$ maps $L^{\infty}(a, b)$ into $C[a, b]$ and is compact between these spaces.

In the sequel, there will be many quotings to Lemma 1.7, not only in the proof of Theorems 1.2 and 1.3 but also in the proof of Lemmas 1.8 and 1.9.

Lemma 1.8. Let $K \in \mathcal{W}^{m, \nu ; \lambda, \mu}((a, b) \times(a, b))$ with parameters $m, \nu, \lambda, \mu$ satisfying (1.8). Then the following is true:

(i) $T_{K}: C^{m, \nu+\lambda, \nu+\mu}(a, b) \rightarrow C^{m, \nu+\lambda, \nu+\mu}(a, b)$ is compact for $\nu \notin \mathbb{Z}$;

(ii) $T_{K}: C_{\star}^{m, \nu+\lambda, \nu+\mu}(a, b) \rightarrow C_{\star}^{m, \nu+\lambda, \nu+\mu}(a, b)$ is compact (for $\nu \in \mathbb{Z}$ as well as for $\nu \notin \mathbb{Z})$.

Lemma 1.9. Let $K \in \mathcal{W}_{\star}^{m, \nu ; \lambda, \mu}((a, b) \times(a, b))$ where the parameters $m, \nu, \lambda$, $\mu$ satisfy (1.8) and $\nu \in \mathbb{Z}$. Then $T_{K}: C^{m, \nu+\lambda, \nu+\mu}(a, b) \rightarrow C^{m, \nu+\lambda, \nu+\mu}(a, b)$ is compact.

Theorem 1.2 immediately follows from Lemmas 1.6-1.8, whereas Theorem 1.3 follows from Lemmas 1.6, 1.7 and 1.9. The proof of Lemma 1.7 is elementary and it is presented in Section 2. The proof of Lemmas 1.8 and 1.9 is a more serious task, we present it in the course of Sections 3-5.

Remark 1.10. Also other reference spaces rather than $F=C[a, b]$ can be used in smoothness results like Theorems 1.1-1.3. According to Lemma 1.6, a sufficient condition on the Banach space $F$ for a modifying of Theorems 1.1-1.3 reads as follows: $C^{m, \nu+\lambda, \nu+\mu}(a, b) \subset F$ densely and continuously, $T$ is compact in $F$. For instance, for $\lambda \leq 0, \mu \leq 0$, the space $F=L^{1}(a, b)$ is suitable whereas in the case of arbitrary $\lambda$ and $\mu$, the weighted space $F=L^{1, \lambda_{+}, \mu_{+}}(a, b)$ equipped with the norm $\int_{a}^{b}|u(y)|(y-a)^{-\lambda_{+}}(b-y)^{-\mu_{+}} d y$ may be used; here $\lambda_{+}=\max \{\lambda, 0\}, \mu_{+}=\max \{\mu, 0\}$.

1.6. Application to Volterra equations. The Volterra integral equation

$$
u(x)=\int_{a}^{x} K(x, y) u(y) d y+f(x), \quad a<x<b,
$$

can be considered as the Fredholm integral equation (1.1) in which $K(x, y)=0$ for $a<x<y<b$. The classes $\mathcal{W}^{m, \nu}((a, b) \times(a, b)), \mathcal{W}^{m, \nu ; \lambda, \mu}((a, b) \times(a, b))$ and 
$\mathcal{W}_{\star}^{m, \nu ; \lambda, \mu}((a, b) \times(a, b))$ have sense for such kernels and Theorems 1.1-1.3 hold for equation (1.11). These results can be specified if $f(x)$ has no singularity at $x=b$ and $K(x, y)$ has no singularity at $y=b$, since then also the solution $u(x)$ of (1.11) has no singularity at $x=b$. Denote

$$
\triangle=\triangle_{a, b}=\{(x, y): a<y<x \leq b\}
$$

and introduce the following classes of kernels for equation (1.11):

$\mathcal{W}^{m, \nu}(\triangle)$ consists of $m$ times continuously differentiable functions $K$ on $\triangle$ that satisfy there for all $k, l, k+l \leq m$, the inequality (1.2);

$\mathcal{W}^{m, \nu ; \lambda}(\triangle)$ consists of $m$ times continuously differentiable functions $K$ on $\triangle$ that satisfy there for all $k, l, k+l \leq m$, the inequality

$$
\left|\left(\frac{\partial}{\partial x}\right)^{k}\left(\frac{\partial}{\partial x}+\frac{\partial}{\partial y}\right)^{l} K(x, y)\right| \leq c_{m, K} \kappa_{\nu+k}(|x-y|)(y-a)^{-\lambda-l}
$$

and $\lim _{y \rightarrow x}\left(\frac{\partial}{\partial x}\right)^{k} K(x, y)=0$ if $\nu+k<0, k \leq m$;

$\mathcal{W}_{\star}^{m, \nu ; \lambda}(\triangle)$ consists of $K \in W^{m, \nu ; \lambda}(\Delta)$ that in addition to (1.12) satisfy

$$
\left|\left(\frac{\partial}{\partial x}\right)^{k}\left(\frac{\partial}{\partial x}+\frac{\partial}{\partial y}\right)^{l} K(x, y)\right| \leq c_{m, K} \kappa_{\nu+k}(|x-y|) \frac{(y-a)^{-\lambda-l}}{1+|\log (y-a)|} \quad \text { if } \lambda+l>0 .
$$

We modify also the weighted spaces: $C^{m, s}(a, b]$ and $C_{\star}^{m, s}(a, b]$ consist of $m$ times continuously differentiable functions $u$ on $(a, b]$ that have a finite norm

$$
\|u\|_{m, s}=\sum_{k=0}^{m} \sup _{a<x \leq b} w_{k+s-1}(x-a)\left|u^{(k)}(x)\right|
$$

and

$$
\|u\|_{m, s}^{\star}=\sum_{k=0}^{m} \sup _{a<x \leq b} w_{k+s-1}^{\star}(x-a)\left|u^{(k)}(x)\right|,
$$

respectively. The specifications of Theorems 1.1-1.3 read as follows.

Theorem 1.11. Let $K \in \mathcal{W}^{m, \nu}(\triangle)$ and $f \in C^{m, \nu}(a, b]$ where $m \geq 1, \nu<1$. Then equation (1.11) has a unique solution and it belongs to $C^{m, \nu}(a, b]$.

Theorem 1.12. Let $K \in \mathcal{W}^{m, \nu ; \lambda}(\triangle)$ where $m \geq 1, \nu<1, \lambda<\min \{1,1-\nu\}$. Then equation (1.11) has a unique solution $u$ and the following is true:

(i) if $\nu \notin \mathbb{Z}$ and $f \in C^{m, \nu+\lambda}(a, b]$, then $u \in C^{m, \nu+\lambda}(a, b]$;

(ii) if $f \in C_{\star}^{m, \nu+\lambda}(a, b]$, then $u \in C_{\star}^{m, \nu+\lambda}(a, b]$ (for $\nu \in \mathbb{Z}$ as well as for $\left.\nu \notin \mathbb{Z}\right)$.

Theorem 1.13. Let $K \in \mathcal{W}_{\star}^{m, \nu ; \lambda}(\triangle), f \in C^{m, \nu+\lambda}(a, b]$ where $m \geq 1,1>\nu \in$ $\mathbb{Z}, \lambda<\min \{1,1-\nu\}$. Then equation (1.11) has a unique solution and it belongs to $C^{m, \nu+\lambda}(a, b]$. 
Theorem 1.11 is known, see [5] where even a nonlinear problem has been considered. Theorems 1.12 and 1.13 are consequences of Theorems 1.2 and 1.3 and a prolongation argument. Namely, we first extend $f$ from $(a, b]$ to $(a, b+\delta]$, $0<\delta<\frac{b-a}{m}$, using the reflection formula (see, e.g., [12])

$$
f(x)=\sum_{j=0}^{m} d_{j} f(b-j(x-b)), \quad b<x \leq b+\delta,
$$

where $d_{j}$ are chosen so that the $C^{m}$-smooth joining happens at $x=b$ :

$$
\sum_{j=0}^{m}(-j)^{k} d_{j}=1, \quad k=0,1, \ldots, m
$$

Using $(1.13),(1.14)$ we also extend $K$ from $\triangle_{a, b}$ to $\triangle_{a, b+\delta}$ along the lines $y-a=$ $\gamma(x-a), 0<\gamma<1$. The extension procedure preserves $f$ in $C^{m, \nu}(a, b+\delta]$ and $K$ in $\mathcal{W}^{m, \nu ; \lambda}\left(\triangle_{a, b+\delta}\right)$ or in the corresponding $\star$-labelled classes. After that we apply Theorems 1.2 and 1.3 to the prolonged problem (1.11) for $a<x<b+\delta$ to be sure that no singularity of the solution at $x=b$ appears.

1.7. Boundary singularities of the kernels with respect to $x$ and $y$. The kernel classes $\mathcal{W}^{m, \nu ; \lambda, \mu}$ and $\mathcal{W}_{\star}^{m, \nu ; \lambda, \mu}$ admit boundary singularities of $K(x, y)$ with respect to $y$ but not with respect to $x$. Here we demonstrate how to treat the integral equations with kernels that have boundary singularities with respect to both arguments. For the brevity we confine ourselves to the problem

$$
u(x)=\int_{a}^{b}(x-a)^{-\lambda_{1}}(b-x)^{-\mu_{1}} K(x, y) u(y) d y+(x-a)^{-\lambda_{1}}(b-x)^{-\mu_{1}} f(x),
$$

$a<x<b$, where $\lambda_{1}$ and $\mu_{1}$ are real parameters, $K \in \mathcal{W}^{m, \nu ; \lambda, \mu}((a, b) \times(a, b))$ and $f \in C^{m, \nu+\lambda+\lambda_{1}, \nu+\mu+\mu_{1}}(a, b)$. With respect to the unknown function

$$
v(x)=(x-a)^{\lambda_{1}}(b-x)^{\mu_{1}} u(x),
$$

equation (1.15) takes the form

$$
v(x)=\int_{a}^{b} K(x, y)(y-a)^{-\lambda_{1}}(b-y)^{-\mu_{1}} v(y) d y+f(x) .
$$

This is an equation of type (1.1) with the kernel $\bar{K}(x, y)=K(x, y)(y-a)^{-\lambda_{1}}(b-$ $y)^{-\mu_{1}}$ which has boundary singularities only with respect to $y$. Moreover, $K \in \mathcal{W}^{m, \nu ; \lambda, \mu}$ implies $\bar{K} \in \mathcal{W}^{m, \nu ; \lambda+\lambda_{1}, \mu+\mu_{1}}$, so we may apply Theorem 1.2 to 
equation (1.17). Under conditions $m \geq 1, \nu<1, \lambda+\lambda_{1}<\min \{1,1-\nu\}$, $\mu+\mu_{1}<\min \{1,1-\nu\}$, we obtain for the solution $v$ of equation (1.17) that

$$
\begin{array}{ll}
v \in C^{m, \nu+\lambda+\lambda_{1}, \nu+\mu+\mu_{1}}(a, b) & \text { if } \nu \notin \mathbb{Z} \\
v \in C_{\star}^{m, \nu+\lambda+\lambda_{1}, \nu+\mu+\mu_{1}}(a, b) & \text { if } \nu \in \mathbb{Z} .
\end{array}
$$

From (1.16), (1.18), (1.19) we can determine the boundary singularities of the solution $u$ to equation (1.15). Also Theorem 1.3 can be applied to equation (1.17) assuming that $\bar{K} \in \mathcal{W}_{\star}^{m, \nu ; \lambda+\lambda_{1}, \mu+\mu_{1}}$; for $\lambda_{1} \leq 0, \mu_{1} \leq 0$, this inclusion is a consequence of the inclusion $K \in \mathcal{W}_{\star}^{m, \nu ; \lambda, \mu}$.

Similarly, the Volterra integral equation

$$
u(x)=\int_{a}^{x}(x-a)^{-\lambda_{1}} K(x, y) u(y) d y+(x-a)^{-\lambda_{1}} f(x), \quad a<x<b,
$$

with $K \in \mathcal{W}^{m, \nu ; \lambda}(\triangle), f \in C^{m, \nu+\lambda+\lambda_{1}}(a, b]$ can be reduced to equation of the type (1.11) with the kernel $\bar{K}(x, y)=K(x, y)(y-a)^{-\lambda_{1}}$ of the class $\mathcal{W}^{m, \nu ; \lambda+\lambda_{1}}(\triangle)$, and Theorems 1.12 and 1.13 can be applied.

\section{Compactness of $T_{K}: L^{\infty}(a, b) \rightarrow C[a, b]$}

Here we prove Lemma 1.7. To this end, we first establish an estimate for the integrals of the type $\int_{x_{1}}^{x_{2}}|K(x, y)| d y$.

Lemma 2.1. Let $K$ satisfy the conditions of Lemma 1.7. Then for any $x_{1}, x_{2} \in$ $[a, b], x_{1}<x_{2}$, there holds

$$
\sup _{a \leq x \leq b} \int_{x_{1}}^{x_{2}}|K(x, y)| d y \leq c_{K} \begin{cases}\left(x_{2}-x_{1}\right)^{\min \{1,1-\lambda, 1-\mu\}}, & \nu<0 \\ c_{\varepsilon}\left(x_{2}-x_{1}\right)^{\min \{1-\varepsilon, 1-\varepsilon-\lambda, 1-\varepsilon-\mu\}}, & \nu=0 \\ \left(x_{2}-x_{1}\right)^{\min \{1-\nu, 1-\nu-\lambda, 1-\nu-\mu\}}, & 0<\nu<1\end{cases}
$$

where in case $\nu=0$ the parameter $\varepsilon \in(0, \min \{1-\lambda, 1-\mu\})$ may be chosen arbitrarily and $c_{\varepsilon}=c_{\varepsilon, b-a}=\sup _{0<r \leq b-a} r^{\varepsilon}(1+|\log r|)$.

Proof. Introduce a cutting function $\sigma \in C[a, b]$ with the properties

$$
\begin{aligned}
0 \leq \sigma(y) \leq 1 & \text { for } a \leq y \leq b \\
\sigma(y)=1 & \text { for } a \leq y \leq a+\frac{1}{3}(b-a) \\
\sigma(y)=0 & \text { for } a+\frac{2}{3}(b-a) \leq y \leq b .
\end{aligned}
$$

Denote $K^{-}(x, y)=K(x, y) \sigma(y)$ and $K^{+}(x, y)=K(x, y)(1-\sigma(y))$. Due to (1.9), $\left|K^{-}(x, y)\right| \leq c \kappa_{\nu}(|x-y|)(y-a)^{-\lambda}$ and $\left|K^{+}(x, y)\right| \leq c \kappa_{\nu}(|x-y|)(b-y)^{-\mu}$. To 
prove (2.1), it is sufficient to establish that

$$
\begin{aligned}
& \sup _{a \leq x \leq b} \int_{x_{1}}^{x_{2}}\left|K^{-}(x, y)\right| d y \leq c \begin{cases}\left(x_{2}-x_{1}\right)^{\min \{1,1-\lambda\}}, & \nu<0 \\
c_{\varepsilon}\left(x_{2}-x_{1}\right)^{\min \{1-\varepsilon, 1-\varepsilon-\lambda,\}}, & \nu=0 \\
\left(x_{2}-x_{1}\right)^{\min \{1-\nu, 1-\nu-\lambda\}}, & 0<\nu<1\end{cases} \\
& \sup _{a \leq x \leq b} \int_{x_{1}}^{x_{2}}\left|K^{+}(x, y)\right| d y \leq c \begin{cases}\left(x_{2}-x_{1}\right)^{\min \{1,1-\mu\}}, & \nu<0 \\
c_{\varepsilon}\left(x_{2}-x_{1}\right)^{\min \{1-\varepsilon, 1-\varepsilon-\mu\}}, & \nu=0 \\
\left(x_{2}-x_{1}\right)^{\min \{1-\nu, 1-\nu-\mu\}}, & 0<\nu<1 .\end{cases}
\end{aligned}
$$

We prove (2.3); the second inequality, (2.4), follows by the symmetry argument. We treat the cases $\nu<0, \nu=0$ and $0<\nu<1$ separately.

In the case $\nu<0$ we have $\kappa_{\nu}(|x-y|) \equiv 1$, and for $x \in[a, b]$,

$$
\begin{aligned}
\int_{x_{1}}^{x_{2}}\left|K^{-}(x, y)\right| d y & \leq c \int_{x_{1}}^{x_{2}}(y-a)^{-\lambda} d y \\
& \leq c^{\prime} \begin{cases}x_{2}-x_{1}, & \lambda \leq 0 \\
\left(x_{2}-a\right)^{1-\lambda}-\left(x_{1}-a\right)^{1-\lambda}, & 0<\lambda<1\end{cases}
\end{aligned}
$$

and (2.3) follows.

In the case $0<\nu<1$ we have $\kappa_{\nu}(|x-y|)=|x-y|^{-\nu}$, and for $x \in[a, b]$,

$$
\int_{x_{1}}^{x_{2}}\left|K^{-}(x, y)\right| d y \leq c \int_{x_{1}}^{x_{2}}|x-y|^{-\nu}(y-a)^{-\lambda} d y .
$$

If $\lambda \leq 0$ we can continue

$$
\int_{x_{1}}^{x_{2}}\left|K^{-}(x, y)\right| d y \leq c \int_{x_{1}}^{x_{2}}|x-y|^{-\nu} d y \leq c^{\prime}\left(x_{2}-x_{1}\right)^{1-\nu},
$$

where the constant $c^{\prime}$ is independent of $x$. If $\lambda>0$ we use the well known inequality $s t \leq \frac{s^{p}}{p}+\frac{t^{q}}{q}$ for $s, t \in \mathbb{R}_{+}, p>1, \frac{1}{p}+\frac{1}{q}=1$. With $s=|x-y|^{-\nu}$, $t=(y-a)^{-\lambda}, p=\frac{\nu+\lambda}{\nu}, q=\frac{\nu+\lambda}{\lambda}$ it yields

$$
|x-y|^{-\nu}(y-a)^{-\lambda} \leq \frac{\nu}{\nu+\lambda}|x-y|^{-\nu-\lambda}+\frac{\lambda}{\nu+\lambda}(y-a)^{-\nu-\lambda}
$$

and

$$
\begin{aligned}
\int_{x_{1}}^{x_{2}}\left|K^{-}(x, y)\right| d y & \leq c \int_{x_{1}}^{x_{2}}\left(\frac{\nu}{\nu+\lambda}|x-y|^{-\nu-\lambda}+\frac{\lambda}{\nu+\lambda}(y-a)^{-\nu-\lambda}\right) d y \\
& \leq c^{\prime}\left(x_{2}-x_{1}\right)^{1-\nu-\lambda}
\end{aligned}
$$

where the constant $c^{\prime}$ is independent of $x \in[a, b]$. This completes the proof of $(2.3)$ in the case $0<\nu<1$. 
Finally, in the case $\nu=0$ we estimate

$$
\kappa_{\nu}(|x-y|)=1+|\log | x-y|| \leq c_{\varepsilon}|x-y|^{-\varepsilon}, \quad 0<\varepsilon<\min \{1,1-\lambda, 1-\mu\},
$$

and obtain (2.3) for $\nu=0$ from the case $0<\nu<1$.

Proof of Lemma 1.7. Having inequality (2.1), the proof of Lemma 1.7 is a simple task. First of all, (2.1) with $x_{1}=a, x_{2}=b$ tells us that $T_{K}$ is bounded in the space $L^{\infty}(a, b):\left\|T_{K}\right\|_{L^{\infty}(a, b) \rightarrow L^{\infty}(a, b)}=\sup _{a<x<b} \int_{a}^{b}|K(x, y)| d y<\infty$. Take a cutting function $\tau \in C[0, \infty)$ such that

$$
\begin{aligned}
0 \leq \tau(r) \leq 1 & \text { for } r \geq 0 \\
\tau(r)=0 & \text { for } 0 \leq r \leq \frac{1}{2} \\
\tau(r)=1 & \text { for } r \geq 1,
\end{aligned}
$$

and introduce for $n=1,2, \ldots$ the kernels

$$
K_{n}(x, y)=\tau(n(y-a)) \tau(n(b-y)) \tau(n(|x-y|)) K(x, y), \quad a \leq x, y \leq b .
$$

The kernel $K_{n}(x, y)$ is continuous on $[a, b] \times[a, b]$, hence the corresponding integral operator $T_{K_{n}}$ maps $L^{\infty}(a, b)$ into $C[a, b]$ and the mapping $T_{K_{n}}: L^{\infty}(a, b) \rightarrow$ $C[a, b]$ is compact. Further, due to $(2.1)$,

$$
\begin{aligned}
\| T_{K}- & T_{K_{n}} \|_{L^{\infty}(a, b) \rightarrow L^{\infty}[a, b]} \\
& =\sup _{a \leq x \leq b} \int_{a}^{b}\left|K(x, y)-K_{n}(x, y)\right| d y \\
& \leq \sup _{a \leq x \leq b}\left(\int_{a}^{a+\frac{1}{n}}+\int_{b-\frac{1}{n}}^{b}+\int_{\max \left\{a, x-\frac{1}{n}\right\}}^{\min \left\{x+\frac{1}{n}, b\right\}}\right)|K(x, y)| d y \rightarrow 0 \quad \text { as } n \rightarrow \infty .
\end{aligned}
$$

Hence, for $u \in L^{\infty}(a, b)$, the function $v=T_{K} u$ lives in $C[a, b]$ as the uniform limit of the continuous functions $v_{n}=T_{K_{n}} u$. Moreover, $T_{K}: L^{\infty}(a, b) \rightarrow C[a, b]$ is compact as the operator norm limit of compact operators $T_{K_{n}}: L^{\infty}(a, b) \rightarrow$ $C[a, b]$.

Remark 2.2. If $K \in \mathcal{W}^{0, \nu ; \lambda, \mu}((a, b) \times(a, b))$ with $\nu<1, \lambda, \mu<\min \{1,1-\nu\}$ then $T_{K}$ maps $L^{\infty}(a, b)$ into $B C(a, b)$ and is bounded between these spaces. (The difference with Lemma 1.7 is in the relaxed continuity condition.)

Remark 2.3. Assume (1.9) with $\nu<1, \lambda<1, \mu<1$ (but not necessarily $\nu+\lambda<1, \nu+\mu<1$ as in (1.10)). Then for $a<x<b$,

$$
\begin{aligned}
& \int_{a}^{b}|K(x, y)| d y \\
& \quad \leq c\left\{\begin{array}{ll}
1, & \nu+\lambda<1 \\
1+|\log (x-a)|, & \nu+\lambda=1 \\
(x-a)^{1-\nu-\lambda}, & \nu+\lambda>1
\end{array}\right\}+c\left\{\begin{array}{ll}
1, & \nu+\mu<1 \\
1+|\log (b-x)|, & \nu+\mu=1 \\
(b-x)^{1-\nu-\mu}, & \nu+\mu>1
\end{array}\right\} .
\end{aligned}
$$




\section{Equivalent norms of $C^{m, s, t}(a, b)$ and $C_{\star}^{m, s, t}(a, b)$}

In the proof of Lemmas 1.8 and 1.9 we use simplified norms of $C^{m, s, t}(a, b)$ and $C_{\star}^{m, s, t}(a, b)$ which are equivalent to the basic norms (1.5) and (1.6).

Lemma 3.1. Let $m \geq 1, s, t \in \mathbb{R}$. For $u \in C^{m, s, t}(a, b), k=0,1, \ldots, m-1$, there holds

$$
\sup _{a<x<b} w_{k+s-1, k+t-1}(x)\left|u^{(k)}(x)-u^{(k)}\left(x_{0}\right)\right| \leq c \sup _{a<x<b} w_{k+s, k+t}(x)\left|u^{(k+1)}(x)\right|,
$$

where $x_{0}$ is a fixed point of $(a, b)$, e.g., $x_{0}=\frac{a+b}{2}$.

The proof is straightforward and omitted. Introduce the seminorms

$$
\begin{aligned}
|u|_{k, s, t} & =\sup _{a<x<b} w_{k+s-1, k+t-1}(x)\left|u^{(k)}(x)\right| \\
|u|_{k, s, t}^{\star} & =\sup _{a<x<b} w_{k+s-1, k+t-1}^{\star}(x)\left|u^{(k)}(x)\right|, \quad k=0,1, \ldots, m .
\end{aligned}
$$

Thus the norms (1.5) and (1.6) can be written in the form $\|u\|_{m, s, t}=\sum_{k=0}^{m}|u|_{k, s, t}$ and $\|u\|_{m, s, t}^{\star}=\sum_{k=0}^{m}|u|_{k, s, t}^{\star}$. With the help of Lemma 3.1 and a similar result for $u \in C_{*}^{m, s, t}(a, b)$ we can prove the following results.

Lemma 3.2. For $m \geq 1, s, t \in \mathbb{R}$, the basic norm $\|u\|_{m, s, t}$ of $C^{m, s, t}(a, b)$ defined in (1.5) is equivalent to the norms

$$
\|u\|_{m, s, t}^{\prime}=\max _{a^{\prime} \leq x \leq b^{\prime}}|u(x)|+|u|_{m, s, t} \quad \text { and } \quad\|u\|_{m, s, t}^{\prime \prime}=\max _{i=1, \ldots, m}\left|u\left(x_{i}\right)\right|+|u|_{m, s, t},
$$

where $\left[a^{\prime}, b^{\prime}\right] \subset(a, b)$ is an arbitrary closed subinterval and $x_{1}, \ldots, x_{m}$ are arbitrary $m$ points of it, $a^{\prime} \leq x_{1}<\ldots<x_{m} \leq b^{\prime}$.

Lemma 3.3. For $m \geq 1, s, t \in \mathbb{R}$, the basic norm $\|u\|_{m, s, t}^{\star}$ of $C_{\star}^{m, s, t}(a, b)$ defined in (1.6) is equivalent to the norms

$$
\|u\|_{m, s, t}^{\star \prime}=\max _{a^{\prime} \leq x \leq b^{\prime}}|u(x)|+|u|_{m, s, t}^{\star} \quad \text { and } \quad\|u\|_{m, s, t}^{\star \prime \prime}=\max _{i=1, \ldots, m}\left|u\left(x_{i}\right)\right|+|u|_{m, s, t}^{\star},
$$

where $\left[a^{\prime}, b^{\prime}\right] \subset(a, b)$ is an arbitrary closed subinterval and $x_{1}, \ldots, x_{m}$ are arbitrary $m$ points of it.

Lemma 3.4. Let $m \geq 1$. The following conditions (i) and (ii) are equivalent for a set $\mathcal{M} \subset C^{m, s, t}(a, b)$ :

(i) $\mathcal{M}$ is relatively compact in $C^{m, s, t}(a, b)$;

(ii) the functions $v$ from $\mathcal{M}$ are $m$ times continuously differentiable in $(a, b)$, uniformly bounded on a subinterval $\left[a^{\prime}, b^{\prime}\right] \subset(a, b)$ (or at least at $m$ points $\left.x_{1}, \ldots, x_{m} \in(a, b)\right)$, and the set $\left\{w_{m+s-1, m+t-1} v^{(m)}: v \in \mathcal{M}\right\}$ is relatively compact in $B C(a, b)$. 
Similarly, the following conditions (i') and (ii') are equivalent for a set $\mathcal{M} \subset$ $C_{\star}^{m, s, t}(a, b)$ :

(i') $\mathcal{M}$ is relatively compact in $C_{\star}^{m, s, t}(a, b)$;

(ii') the functions $v$ from $\mathcal{M}$ are $m$ times continuously differentiable in $(a, b)$, uniformly bounded on a subinterval $\left[a^{\prime}, b^{\prime}\right] \subset(a, b)$ (or at least at $m$ points $\left.x_{1}, \ldots, x_{m} \in(a, b)\right)$, and the set $\left\{w_{m+s-1, m+t-1}^{\star} v^{(m)}: v \in \mathcal{M}\right\}$ is relatively compact in $B C(a, b)$.

Proof. These claims are obvious consequences of Lemmas 3.2 and 3.3.

\section{Differentiation of weakly singular integrals}

First we recall a well known result about the closedness of the graph of the differentiation operator.

Lemma 4.1. Let $v_{n} \in C^{1}(a, b), v_{n} \rightarrow v, v_{n}^{\prime} \rightarrow w$ uniformly on every closed subinterval $\left[a^{\prime}, b^{\prime}\right] \subset(a, b)$. Then $v \in C^{1}(a, b)$ and $v^{\prime}=w$.

The following differentiation result is also known at least partly, see [22][24]. We equip it with an elementary proof based on Lemma 4.1.

Lemma 4.2. Assume that $g(x, y)$ is a continuously differentiable function on $((a, b) \times[a, b]) \backslash \operatorname{diag}$ and satisfies with a $\nu \in(0,1)$ the inequalities

$$
|g(x, y)| \leq c|x-y|^{-\nu} \quad \text { and } \quad\left|\left(\frac{\partial}{\partial x}+\frac{\partial}{\partial y}\right) g(x, y)\right| \leq c|x-y|^{-\nu}
$$

Then the function $\int_{a}^{b} g(x, y) d y$ is continuously differentiable in $(a, b)$ and

$$
\frac{d}{d x} \int_{a}^{b} g(x, y) d y=\int_{a}^{b}\left(\frac{\partial}{\partial x}+\frac{\partial}{\partial y}\right) g(x, y) d y+g(x, a)-g(x, b) .
$$

Proof. For functions $g$ that are continuously differentiable on $(a, b) \times[a, b]$, including the diagonal, formula (4.2) is obvious. Let $g$ satisfy the conditons of the lemma. Take a cutting function $\tau \in C^{1}[0, \infty)$ that satisfies $(2.5)$, and define $g_{n}(x, y)=\tau(n|x-y|) g(x, y), n=1,2, \ldots$ The functions $g_{n}$ are continuously differentiable on $(a, b) \times[a, b]$ and equality (4.2) holds for them true: denoting $v_{n}(x)=\int_{a}^{b} \tau(n|x-y|) g(x, y) d y$, we have

$$
\begin{aligned}
v_{n}^{\prime}(x)= & \frac{d}{d x} \int_{a}^{b} \tau(n|x-y|) g(x, y) d y \\
= & \int_{a}^{b} \tau(n|x-y|)\left(\frac{\partial}{\partial x}+\frac{\partial}{\partial y}\right) g(x, y) d y \\
& +\tau(n(x-a)) g(x, a)-\tau(n(b-x)) g(x, b), \quad a<x<b .
\end{aligned}
$$


We took into account that $\left(\frac{\partial}{\partial x}+\frac{\partial}{\partial y}\right) \tau(n|x-y|)=0$. With the help of (4.1) we find that

$$
v_{n}^{\prime}(x) \rightarrow \int_{a}^{b}\left(\frac{\partial}{\partial x}+\frac{\partial}{\partial y}\right) g(x, y) d y+g(x, a)-g(x, b), \quad v_{n}(x) \rightarrow \int_{a}^{b} g(x, y) d y
$$

as $n \rightarrow \infty$ uniformly on every closed subinterval $\left[a^{\prime}, b^{\prime}\right] \subset(a, b)$. By Lemma 4.1, the function $\int_{a}^{b} g(x, y) d y$ is continuously differentiable on $(a, b)$ and (4.2) holds for it.

We are ready to derive a formula for the differentiation of $T_{K} u$. Assume that $K$ satifies the conditions of Lemma 1.8, i.e.,

$$
K \in \mathcal{W}^{m, \nu ; \lambda, \mu}((a, b) \times(a, b)), \quad m \geq 1, \nu<1, \quad \lambda, \mu<\min \{1,1-\nu\},
$$

and take an arbitrary function

$$
u \in C^{m, \nu+\lambda, \nu+\mu}(a, b) \text { or } \quad u \in C_{\star}^{m, \nu+\lambda, \nu+\mu}(a, b), \quad \lambda, \mu<\min \{1,1-\nu\} .
$$

Denote by $k^{\prime} \geq 0$ be the greatest integer that is less than $1-\nu$, i.e.,

$$
k^{\prime}=[1-\nu] \quad \text { for } \nu \notin \mathbb{Z}, \quad k^{\prime}=-\nu \quad \text { for } \nu \in \mathbb{Z},
$$

where $[1-\nu]$ is the integer part of $1-\nu$. In particular, $k^{\prime}=0$ in the most interestig cases where $0 \leq \nu<1$. We assume now that $m>k^{\prime}$ (as we will see, the case $m \leq k^{\prime}$ is trivial). Due to condition (1.3) with $l=0$, the kernel $\left(\frac{\partial}{\partial x}\right)^{k^{\prime}} K(x, y)$ is still weakly singular and we may compute $\left(\frac{d}{d x}\right)^{k^{\prime}}\left(T_{K} u\right)(x)$ by differentiating the kernel under the integral,

$$
\left(\frac{d}{d x}\right)^{k^{\prime}}\left(T_{K} u\right)(x)=\int_{a}^{b}\left(\frac{\partial}{\partial x}\right)^{k^{\prime}} K(x, y) u(y) d y
$$

recall that $\left(\frac{\partial}{\partial x}\right)^{k} K(x, y)$ is continuous on $(a, b) \times(a, b)$ for $k<k^{\prime}$. To compute

$$
\left(\frac{d}{d x}\right)^{m}\left(T_{K} u\right)(x)=\left(\frac{d}{d x}\right)^{m-k^{\prime}} \int_{a}^{b}\left(\frac{\partial}{\partial x}\right)^{k^{\prime}} K(x, y) u(y) d y
$$

we take a cutting function $\tau \in C^{m}[0, \infty)$ that satisfies (2.5). Fix an arbitrary point $x^{\prime} \in(a, b)$ and denote $r^{\prime}=\frac{1}{2} \rho\left(x^{\prime}\right)$ where $\rho(x)=\min \{x-a, b-x\}$ is the distance from $x \in(a, b)$ to the boundary of $(a, b)$. For $\left|x-x^{\prime}\right| \leq \frac{1}{2} r^{\prime}$, we represent

$$
\begin{aligned}
\int_{a}^{b}\left(\frac{\partial}{\partial x}\right)^{k^{\prime}} K(x, y) u(y) d y= & \int_{a}^{b} \tau\left(\frac{|x-y|}{r^{\prime}}\right)\left(\frac{\partial}{\partial x}\right)^{k^{\prime}} K(x, y) u(y) d y \\
& +\int_{a}^{b}\left\{1-\tau\left(\frac{|x-y|}{r^{\prime}}\right)\right\}\left(\frac{\partial}{\partial x}\right)^{k^{\prime}} K(x, y) u(y) d y
\end{aligned}
$$


The singularity at $x=y$ is cut off in the first integral on r.h.s., and we may apply $\left(\frac{\partial}{\partial x}\right)^{m-k^{\prime}}$ under the integral. In the second integral on r.h.s., the coefficient function $1-\tau\left(\frac{1}{r^{\prime}}|x-y|\right)$ vanishes for $|x-y| \geq r^{\prime}$, in particular, for $y$ satisfying $\left|y-x^{\prime}\right| \geq \frac{3}{2} r^{\prime}$ (since $\left|x-x^{\prime}\right| \leq \frac{1}{2} r^{\prime}$ ); the boundary points $a$ and $b$ with their $\frac{1}{2} r^{\prime}$-neighbourhoods belong to the region where $1-\tau\left(\frac{1}{r^{\prime}}|x-y|\right)$ vanishes. Thus in the second integral the boundary singularities caused by $K(x, y)$ are cut off. Due to estimate (1.3), differentiation formula (4.2) may be applied obtaining

$$
\begin{aligned}
& \frac{\partial}{\partial x} \int_{a}^{b}\left\{1-\tau\left(\frac{|x-y|}{r^{\prime}}\right)\right\}\left(\frac{\partial}{\partial x}\right)^{k^{\prime}} K(x, y) u(y) d y \\
& \quad=\int_{a}^{b}\left\{1-\tau\left(\frac{|x-y|}{r^{\prime}}\right)\right\}\left(\frac{\partial}{\partial x}+\frac{\partial}{\partial y}\right)\left\{\left(\frac{\partial}{\partial x}\right)^{k^{\prime}} K(x, y) u(y)\right\} d y
\end{aligned}
$$

Recall that $1-\tau\left(\frac{1}{r^{\prime}}|x-y|\right)=0$ for $y=a$ and $y=b$, so the boundary terms of the formula (4.2) vanish in our case; we also took into account that $\left(\frac{\partial}{\partial x}+\frac{\partial}{\partial y}\right) \tau\left(\frac{1}{r^{\prime}}|x-y|\right)=0$. In its turn, the last integral may be differentiated in the similar manner. So for $\left|x-x^{\prime}\right| \leq \frac{r^{\prime}}{2}$ we obtain

$$
\begin{aligned}
& \left(\frac{d}{d x}\right)^{m}\left(T_{K} u\right)(x) \\
& =\int_{a}^{b}\left(\frac{\partial}{\partial x}\right)^{m-k^{\prime}}\left\{\tau\left(\frac{|x-y|}{r^{\prime}}\right)\left(\frac{\partial}{\partial x}\right)^{k^{\prime}} K(x, y)\right\} u(y) d y \\
& \quad+\int_{a}^{b}\left\{1-\tau\left(\frac{|x-y|}{r^{\prime}}\right)\right\}\left(\frac{\partial}{\partial x}+\frac{\partial}{\partial y}\right)^{m-k^{\prime}}\left\{\left(\frac{\partial}{\partial x}\right)^{k^{\prime}} K(x, y) u(y)\right\} d y
\end{aligned}
$$

Differentiating the product of functions by the Leibnitz rule, taking the result at point $x=x^{\prime}$ but writing again $x$ instead of $x^{\prime}$, we arrive at the formula

$$
\begin{aligned}
\left(\frac{d}{d x}\right)^{m}\left(T_{K} u\right)(x)= & \sum_{j=0}^{m-k^{\prime}}\left(\begin{array}{c}
m-k^{\prime} \\
j
\end{array}\right) \int_{a}^{b} \tau_{j}(x, y)\left(\frac{\partial}{\partial x}\right)^{k^{\prime}+j} K(x, y) u(y) d y \\
& +\sum_{j=0}^{m-k^{\prime}}\left(\begin{array}{c}
m-k^{\prime} \\
j
\end{array}\right) \int_{a}^{b}\left\{1-\tau\left(\frac{2|x-y|}{\rho(x)}\right)\right\} \\
& \cdot\left\{\left(\frac{\partial}{\partial x}+\frac{\partial}{\partial y}\right)^{m-k^{\prime}-j}\left(\frac{\partial}{\partial x}\right)^{k^{\prime}} K(x, y)\right\} u^{(j)}(y) d y
\end{aligned}
$$

$a<x<b$, where

$$
\tau_{j}(x, y)=\left[\left(\frac{\partial}{\partial x}\right)^{m-k^{\prime}-j} \tau\left(\frac{|x-y|}{r}\right)\right]_{r=\frac{\rho(x)}{2}}, \quad j=0, \ldots, m-k^{\prime} .
$$

Let us summarise the result. 
Lemma 4.3. For $K$ and $u$ satisfying (4.3) and (4.4) with $m>k^{\prime}$ (where $k^{\prime}$ is defined by (4.5)), the derivative $\left(\frac{d}{d x}\right)^{m}\left(T_{K} u\right)(x)$ exists in $(a, b)$ and can be represented by the formula (4.6) where $\tau_{j}(x, y)$ is defined in (4.7) and the cutting function $\tau \in C^{m}[0, \infty)$ satisfies (2.5). In the case $m \leq k^{\prime}$ we simply have

$$
\left(\frac{d}{d x}\right)^{m}\left(T_{K} u\right)(x)=\int_{a}^{b}\left(\frac{\partial}{\partial x}\right)^{m} K(x, y) u(y) d y, \quad a<x<b .
$$

\section{Compactness of $T_{K}$ in $C^{m, \nu+\lambda, \nu+\mu}(a, b)$ and $C_{\star}^{m, \nu+\lambda, \nu+\mu}(a, b)$}

Let us multiply both sides of (4.6) by the weight function $w_{m+\nu+\lambda-1, m+\nu+\mu-1}$ corresponding to $\left(\frac{\partial}{\partial x}\right)^{m}\left(T_{K} u\right)(x)$. The result can be written in the form

$$
\begin{aligned}
w_{m+\nu+\lambda-1, m+\nu+\mu-1} & D^{m} T_{K} u \\
& =\sum_{j=0}^{m-k^{\prime}}\left(\begin{array}{c}
m-k^{\prime} \\
j
\end{array}\right)\left(T_{j} u+S_{j}\left(w_{j+\nu+\lambda-1, j+\nu+\mu-1} D^{j} u\right)\right)
\end{aligned}
$$

where $D=\frac{d}{d x}$ is the differentiation operator and

$$
\begin{aligned}
\left(T_{j} u\right)(x)= & \int_{a}^{b} w_{m+\nu+\lambda-1, m+\nu+\mu-1}(x) \tau_{j}(x, y)\left(\frac{\partial}{\partial x}\right)^{k^{\prime}+j} K(x, y) u(y) d y \\
\left(S_{j} v\right)(x)= & \int_{a}^{b} \frac{w_{m+\nu+\lambda-1, m+\nu+\mu-1}(x)}{w_{j+\nu+\lambda-1, j+\nu+\mu-1}(y)}\left\{1-\tau\left(\frac{2|x-y|}{\rho(x)}\right)\right\} \\
& \cdot\left\{\left(\frac{\partial}{\partial x}\right)^{k^{\prime}}\left(\frac{\partial}{\partial x}+\frac{\partial}{\partial y}\right)^{m-k^{\prime}-j} K(x, y)\right\} v(y) d y .
\end{aligned}
$$

The proof of Lemmas 1.8 (i) and 1.9 can be reduced to the study of the mapping properties of $T_{j}$ and $S_{j}$. In (5.1), $w_{j+\nu+\lambda-1, j+\nu+\mu-1}(y)=1$ for $j=0$ and

$$
\sup _{a<y<b} w_{j+\nu+\lambda-1, j+\nu+\mu-1}(y)\left|\left(D^{j} u\right)(y)\right| \leq\|u\|_{m, \nu+\lambda, \nu+\mu}, \quad j=0,1, \ldots, m .
$$

Recall that the imbedding $C^{m, \nu+\lambda, \nu+\mu}(a, b) \subset C[a, b]$ is compact. Taking into account also Lemmas 3.2 and 3.4 we observe that in order to prove the compactness of the operator $T_{K}$ in $C^{m, \nu+\lambda, \nu+\mu}(a, b)$, it is sufficient to establish that

$$
\begin{aligned}
S_{0}, T_{j}: B C(a, b) & \rightarrow B C(a, b), \quad j=0,1, \ldots, m-k^{\prime}, & \text { are bounded } \\
S_{j}: B C(a, b) & \rightarrow B C(a, b), \quad j=1, \ldots, m-k^{\prime}, & \text { are compact. }
\end{aligned}
$$

In the sequel we realise (5.4), (5.5) for $\nu \notin \mathbb{Z}$ but for $\nu \in \mathbb{Z}$ we slightly modify the program: while $T_{0}$ occurs to be unbounded in $B C(a, b)$ for $\nu \in \mathbb{Z}$ in general, we 
prove that under condition $K \in \mathcal{W}_{\star}^{m, \nu ; \lambda, \mu}$, neverteless, $T_{0}: C^{m, \nu+\lambda, \nu+\mu}(a, b) \rightarrow$ $B C(a, b)$ is compact.

To prove Lemma 1.8 (ii), we have to examine $T_{K}$ also in the space $C_{\star}^{m, \nu+\lambda, \nu+\mu}(a, b)$. To do this we multiply (4.6) by $w_{m+\nu+\lambda-1, m+\nu+\mu-1}^{\star}$ obtaining the formulae quite similar to (5.1)-(5.3): everywhere the weight functions $w_{j+\nu+\lambda-1, j+\nu+\mu-1}$ are replaced by their counterparts $w_{j+\nu+\lambda-1, j+\nu+\mu-1}^{\star}$. We denote the corresponding integral operators by $T_{j}^{\star}, S_{j}^{\star}, j=0, \ldots, m$. We realise the program like $(5.4),(5.5)$ for $T_{j}^{\star}, S_{j}^{\star}, j=0, \ldots, m$, this time without any exception.

Lemma 5.1. Assume that $K \in \mathcal{W}^{m, \nu ; \lambda, \mu}((a, b) \times(a, b)), m \geq k^{\prime}+1, \nu<1$, $\lambda, \mu<\min \{1,1-\nu\}$. Then the operators $S_{j}$ and $S_{j}^{\star}, j=1, \ldots, m-k^{\prime}$, are compact in the space $B C(a, b)$, i.e., (5.5) holds true (independently of whether $\nu \in \mathbb{Z}$ or $\nu \notin \mathbb{Z}$ ). Further, the operator $S_{0}$ is bounded in $B C(a, b)$ if $\nu \notin \mathbb{Z}$ (and may be unbounded for $\nu \in \mathbb{Z})$ whereas the operator $S_{0}^{\star}$ is bounded in $B C(a, b)$ independently of $\nu$.

If $K \in \mathcal{W}_{\star}^{m, \nu ; \lambda, \mu}((a, b) \times(a, b)), m \geq k^{\prime}+1, \nu<1, \lambda, \mu<\min \{1,1-\nu\}$, then $S_{0}$ is bounded in $B C(a, b)$ also for $\nu \in \mathbb{Z}$.

Proof. Denote by $H_{j}$ the kernel of the integral operator $S_{j}, 0 \leq j \leq m-k^{\prime}$,

$$
\begin{aligned}
H_{j}(x, y)= & \frac{w_{m+\nu+\lambda-1, m+\nu+\mu-1}(x)}{w_{j+\nu+\lambda-1, j+\nu+\mu-1}(y)} \\
& \cdot\left\{1-\tau\left(\frac{2|x-y|}{\rho(x)}\right)\right\}\left(\frac{\partial}{\partial x}\right)^{k^{\prime}}\left(\frac{\partial}{\partial x}+\frac{\partial}{\partial y}\right)^{m-k^{\prime}-j} K(x, y) .
\end{aligned}
$$

For $j=1, \ldots, m-k^{\prime}$ we check that $H_{j}$ is weakly singular and obtain (5.5) on the basis of of Lemma 1.7. The order of derivatives of $K$ involved in $H_{j}$ is $m-j$, and those have a continuous extension to $([a, b] \times(a, b)) \backslash$ diag for $j \geq 1$, hence the same property has $H_{j}$. Estimate (1.3) yields

$$
\begin{aligned}
\mid\left(\frac{\partial}{\partial x}\right)^{k^{\prime}}\left(\frac{\partial}{\partial x}\right. & \left.+\frac{\partial}{\partial y}\right)^{m-k^{\prime}-j} K(x, y) \mid \\
& \leq c \kappa_{\nu+k^{\prime}}(|x-y|)(y-a)^{-\lambda-m+k^{\prime}+j}(b-y)^{-\mu-m+k^{\prime}+j}
\end{aligned}
$$

To separate the boundary singularities, introduce the operators $S_{j}^{-}$and $S_{j}^{+}$with the kernels $H_{j}^{-}(x, y)=H_{j}(x, y) \sigma(y)$ and $H_{j}^{+}(x, y)=H_{j}(x, y)(1-\sigma(y))$, respectively, where the cutting function $\sigma \in C[a, b]$ satisfies (2.2). Since $S_{j}=S_{j}^{-}+S_{j}^{+}$, it is sufficient to establish the claims of the Lemma for $S_{j}^{-}$and $S_{j}^{+}$separately, or thanks to symmetry, for $S_{j}^{-}$only. We have

$$
\begin{aligned}
& \left|H_{j}^{-}(x, y)\right| \\
& \quad \leq c \frac{w_{m+\nu+\lambda-1}(x-a)}{w_{j+\nu+\lambda-1}(y-a)}\left\{1-\tau\left(\frac{2|x-y|}{\rho(x)}\right)\right\} \kappa_{\nu+k^{\prime}}(|x-y|)(y-a)^{-\lambda-m+k^{\prime}+j}
\end{aligned}
$$




$$
\begin{aligned}
& \left|H_{j}^{+}(x, y)\right| \\
& \quad \leq c \frac{w_{m+\nu+\mu-1}(b-x)}{w_{j+\nu+\mu-1}(b-y)}\left\{1-\tau\left(\frac{2|x-y|}{\rho(x)}\right)\right\} \kappa_{\nu+k^{\prime}}(|x-y|)(b-y)^{-\mu-m+k^{\prime}+j} .
\end{aligned}
$$

In the sequel we confine us to the examining of $H_{j}^{-}(x, y), j=0, \ldots, m-k^{\prime}$.

¿From definition of $k^{\prime}$ (see (4.5)) we observe that $\nu+k^{\prime}=0$ for $\nu \in \mathbb{Z}$ and $0<\nu+k^{\prime}<1$ for $\nu \notin \mathbb{Z}$, thus $\kappa_{\nu+k^{\prime}}(|x-y|)$ has at most a weak singularity on the diagonal,

$$
\kappa_{\nu+k^{\prime}}(|x-y|) \leq c \begin{cases}1+|\log | x-y||, & \nu \in \mathbb{Z} \\ |x-y|^{-\nu-k^{\prime}}, & \nu \notin \mathbb{Z} .\end{cases}
$$

Further, $1-\tau\left(\frac{2|x-y|}{\rho(x)}\right)=0$ for $|x-y| \geq \frac{\rho(x)}{2}$, hence the integration interval in (5.3) actually is $\left(x-\frac{\rho(x)}{2}, x+\frac{\rho(x)}{2}\right) \subset(a, b)$. In this subinterval, the quantities $\rho(x)$ and $\rho(y)$ are of the same order, namely,

$$
\frac{\rho(x)}{2} \leq \rho(y) \leq 3 \frac{\rho(x)}{2} \text { for } y \in\left(x-\frac{\rho(x)}{2}, x+\frac{\rho(x)}{2}\right) .
$$

Hence similar relations hold for the weight functions: with some positive constants $c_{1}$ and $c_{2}$,

$c_{1} w_{j+\nu+\lambda-1}(x-a) \leq w_{j+\nu+\lambda-1}(y-a) \leq c_{2} w_{j+\nu+\lambda-1}(x-a), \quad j=0, \ldots, m-k^{\prime}$.

Thus

$$
\left|H_{j}^{-}(x, y)\right| \leq c h_{j}^{-}(x) \kappa_{\nu+k^{\prime}}(|x-y|), \quad j=0, \ldots, m-k^{\prime}
$$

where

$$
h_{j}^{-}(x)=\frac{w_{m+\nu+\lambda-1}(x-a)}{w_{j+\nu+\lambda-1}(x-a)}(x-a)^{-\lambda-m+k^{\prime}+j} .
$$

Depending on the signs of $m+\nu+\lambda-1$ and $j+\nu+\lambda-1$, we have 6 cases to specify $h_{j}^{-}(x)$.

Case 1: $m+\nu+\lambda-1>0, j+\nu+\lambda-1>0$ (in this case $j \geq 1$ since $\lambda<1-\nu)$. Then

$$
\begin{aligned}
h_{j}^{-}(x) & =(x-a)^{m-j}(x-a)^{-\lambda-m+k^{\prime}+j}=(x-a)^{k^{\prime}-\lambda} \\
\left|H_{j}^{-}(x, y)\right| & \leq c(x-a)^{k^{\prime}-\lambda} \kappa_{\nu+k^{\prime}}(|x-y|),
\end{aligned}
$$

or, once more exploiting (5.6), $\left|H_{j}^{-}(x, y)\right| \leq c \kappa_{\nu+k^{\prime}}(|x-y|)(y-a)^{k^{\prime}-\lambda}$, for $(x, y) \in$ $[a, b] \times(a, b) \backslash$ diag. For the singularity orders we have (cf. (1.9), (1.10))

$$
\nu+k^{\prime}<1, \quad-k^{\prime}+\lambda \leq \lambda<1, \quad\left(\nu+k^{\prime}\right)+\left(-k^{\prime}+\lambda\right)=\nu+\lambda<1,
$$


thus $H_{j}^{-}(x, y)$ is a weakly singular kernel satisfying the conditions of Lemma 1.7, and by Lemma $1.7, S_{j}^{-}$is a compact operator in the space $B C(a, b)$.

Case 2: $m+\nu+\lambda-1>0, j+\nu+\lambda-1=0$ (again, $j \geq 1$ in this case). Similarly as in case 1 we find that

$$
\begin{aligned}
h_{j}^{-}(x) & =(1+|\log (x-a)|)(x-a)^{m+\nu+\lambda-1}(x-a)^{-\lambda-m+k^{\prime}+j} \\
& =(1+|\log (x-a)|)(x-a)^{k^{\prime}-\lambda} \\
\left|H_{j}^{-}(x, y)\right| & \leq c_{\varepsilon} \kappa_{\nu+k^{\prime}}(|x-y|)(y-a)^{k^{\prime}-\lambda-\varepsilon},
\end{aligned}
$$

$(x, y) \in[a, b] \times(a, b) \backslash$ diag. We estimated $1+|\log (x-a)| \leq c_{\varepsilon}(x-a)^{-\varepsilon}$ choosing a small $\varepsilon>0$ so that still $\lambda+\varepsilon<1, \lambda+\nu+\varepsilon<1$. Then the conclusions are similar to case 1: $S_{j}^{-}$is compact in the space $B C(a, b)$.

Case 3: $m+\nu+\lambda-1>0, j+\nu+\lambda-1<0$ that implies

$$
\begin{aligned}
h_{j}^{-}(x) & =(x-a)^{m+\nu+\lambda-1}(x-a)^{-\lambda-m+k^{\prime}+j}=(x-a)^{\nu+k^{\prime}+j-1} \\
\left|H_{j}^{-}(x, y)\right| & \leq c \kappa_{\nu+k^{\prime}}(|x-y|)(y-a)^{\nu+k^{\prime}+j-1},
\end{aligned}
$$

$(x, y) \in[a, b] \times(a, b) \backslash$ diag. Now the singularity parameters satisfy

$$
\nu+k^{\prime}<1, \quad-\left(\nu+k^{\prime}+j-1\right) \leq 1-j, \quad\left(\nu+k^{\prime}\right)-\left(\nu+k^{\prime}+j-1\right)=1-j,
$$

and on the bases of Lemma 1.7 , for $j \geq 1$ the operator $S_{j}^{-}$is compact in the space $B C(a, b)$. For $j=0, \nu \notin \mathbb{Z}$, we have on the basis of (5.6)

$$
\sup _{a<x<b} \int_{a}^{b}\left|H_{0}^{-}(x, y)\right| d y \leq c \sup _{a<x<b}(x-a)^{\nu+k^{\prime}-1} \int_{|y-x|<\frac{\rho(x)}{2}}|x-y|^{-\nu-k^{\prime}} d y<\infty
$$

telling us that $S_{0}^{-}$is bounded in $B C(a, b)$. On the other hand, for $j=0, \nu \in \mathbb{Z}$, we have $\nu+k^{\prime}=0$ and

$$
\sup _{a<x<b} \int_{a}^{b}\left|H_{0}^{-}(x, y)\right| d y \leq c \sup _{a<x<b}(x-a)^{-1} \int_{|y-x|<\frac{\rho(x)}{2}}(1+|\log | x-y||) d y=\infty
$$

warning us that $S_{0}^{-}$need not to be bounded in $B C(a, b)$. The situation changes if $K \in \mathcal{W}_{\star}^{m, \nu ; \lambda, \mu}$ : since $\lambda+\left(m-k^{\prime}\right)=m+\nu+\lambda>1$, we may use the estimate (1.4) obtaining for $a \leq y \leq a+\frac{2}{3}(b-a)$ (where $\sigma(y)$ is supported, see the definition of $H_{0}^{-}$)

$$
\left|\left(\frac{\partial}{\partial x}\right)^{k^{\prime}}\left(\frac{\partial}{\partial x}+\frac{\partial}{\partial y}\right)^{m-k^{\prime}} K(x, y)\right| \leq c(1+|\log | x-y||) \frac{(y-a)^{-\lambda-m+k^{\prime}}}{1+|\log (y-a)|} .
$$

Due to (5.6), $1+|\log (y-a)| \asymp 1+|\log (x-a)|$, and

$$
\left|H_{0}^{-}(x, y)\right| \leq c \frac{1}{(x-a)(1+|\log (x-a)|)}(1+|\log | x-y||)
$$


for $(x, y) \in[a, b] \times(a, b) \backslash$ diag that implies the boundedness of $S_{0}^{-}$in $B C(a, b)$ :

$$
\begin{aligned}
& \sup _{a<x \leq x_{0}} \int_{a}^{b}\left|H_{0}^{-}(x, y)\right| d y \\
& \quad \leq c \sup _{a<x<b} \frac{1}{(x-a)(1+|\log (x-a)|)} \int_{|y-x|<\rho(x) / 2}(1+|\log | x-y||) d y<\infty .
\end{aligned}
$$

Case 4: $m+\nu+\lambda-1=0, j+\nu+\lambda-1=0$ (hence $j=m \geq 1$ ). Then $h_{j}^{-}(x)=(x-a)^{-\lambda-m+k^{\prime}+j}=(x-a)^{k^{\prime}-\lambda}$ that is same as in case 1 , and $S_{j}^{-}=S_{m}^{-}$ is compact in $B C(a, b)$.

Case 5: $m+\nu+\lambda-1=0, j+\nu+\lambda-1<0$. Then

$$
\begin{aligned}
h_{j}^{-}(x) & =\frac{1}{1+|\log (x-a)|}(x-a)^{-\lambda-m+k^{\prime}+j} \\
& =\frac{1}{1+|\log (x-a)|}(x-a)^{\nu+k^{\prime}+j-1} \\
\left|H_{j}^{-}(x, y)\right| & \leq c \kappa_{\nu+k^{\prime}}(|x-y|) \frac{(y-a)^{\nu+k^{\prime}+j-1}}{1+|\log (y-a)|},
\end{aligned}
$$

$(x, y) \in[a, b] \times(a, b) \backslash$ diag. This is somewhat stronger estimate than in case 3 due to $1+|\log (y-a)|$ in the denominator. The conclusions are same as in case 3: for $j \geq 1$, the operators $S_{j}^{-}$are compact and $S_{0}^{-}$is bounded in $B C(a, b)$; now even in case $\nu \in \mathbb{Z}$ we do not need the condition $K \in \mathcal{W}_{\star}^{m, \nu ; \lambda, \mu}$ when $S_{0}^{-}$is treated.

Case 6: $m+\nu+\lambda-1<0, j+\nu+\lambda-1<0$. Then

$$
\begin{aligned}
h_{j}^{-}(x) & =(x-a)^{-\lambda-m+k^{\prime}+j} \\
\left|H_{j}^{-}(x, y)\right| & \leq c \kappa_{\nu+k^{\prime}}(|x-y|)(y-a)^{-\lambda-m+k^{\prime}+j},
\end{aligned}
$$

$(x, y) \in[a, b] \times(a, b) \backslash$ diag. In the present case the singularity parameters satisfy strict inequalities

$$
\begin{aligned}
& \nu+k^{\prime}<1 \\
& \lambda+m-k^{\prime}-j=(m+\nu+\lambda-1)-\left(\nu+k^{\prime}\right)-j+1<1-j \\
& \left(\nu+k^{\prime}\right)+\left(\lambda+m-k^{\prime}-j\right)=(m+\nu+\lambda-1)-j+1<1-j,
\end{aligned}
$$

and $S_{j}^{-}: B C(a, b) \rightarrow B C(a, b)$ is by Lemma 1.7 compact for $j \geq 1$ and $S_{0}^{-}$is bounded (for $\nu \in \mathbb{Z}$ as well as for $\nu \notin \mathbb{Z}$ ) .

We completed the proof of claims of Lemma 5.1 concerning the operators $S_{j}$. Now consider the operators $S_{j}^{\star}$ having the kernels

$$
\begin{aligned}
H_{j}^{\star}(x, y)= & \frac{w_{m+\nu+\lambda-1, m+\nu+\mu-1}^{\star}(x\}}{w_{j+\nu+\lambda-1, j+\nu+\mu-1}^{\star}(y)} \\
& \cdot\left\{1-\tau\left(\frac{2|x-y|}{\rho(x)}\right)\right\}\left(\frac{\partial}{\partial x}\right)^{k^{\prime}}\left(\frac{\partial}{\partial x}+\frac{\partial}{\partial y}\right)^{m-k^{\prime}-j} K(x, y) .
\end{aligned}
$$


Separating the boundary singularities similarly as above we arrive at the estimate

$$
\begin{aligned}
\left|H_{j}^{-, \star}(x, y)\right| & \leq c h_{j}^{-, \star}(x) \kappa_{\nu+k^{\prime}}(|x-y|), \quad j=0, \ldots, m-k^{\prime} \\
h_{j}^{-, \star}(x) & =\frac{w_{m+\nu+\lambda-1}^{\star}(x-a)}{w_{j+\nu+\lambda-1}^{\star}(x-a)}(x-a)^{-\lambda-m+k^{\prime}+j}
\end{aligned}
$$

Depending on the signs of $m+\nu+\lambda-1$ and $j+\nu+\mu-1$, we now have 3 different formulae for $h_{j}^{-, \star}(x)$.

Case 1': $m+\nu+\lambda-1 \geq 0, j+\nu+\lambda-1 \geq 0$ (implying $j \geq 1$ ). Then $h_{j}^{-, \star}(x)$ coincides with $h_{j}^{-}(x)$ in case 1 and the result is that $S_{j}^{-, \star}$ is compact in $B C(a, b)$.

Case 2': $m+\nu+\lambda-1 \geq 0, j+\nu+\lambda-1<0$. Then the estimate of $h_{j}^{-, \star}(x)$ is comparable with case 3 but now we have the supplementary logarithm in the denominator. The $S_{j}^{-, \star}, j \geq 1$, are compact in $B C(a, b)$, and $S_{0}^{-, \star}$ is bounded in $B C(a, b)$.

Case 3': $m+\nu+\lambda-1<0, j+\nu+\lambda-1<0$. Then $h_{j}^{-, \star}(x)$ coincides with $h_{j}^{-}(x)$ in case 6 and the result is that $S_{j}^{-, \star}, j \geq 1$, are compact and $S_{0}^{-, \star}$ bounded in $B C(a, b)$.

The proof of Lemma 5.1 is complete.

The last assertion of Lemma 5.1 concerning the boundedness of $S_{0}$ for $\nu \in \mathbb{Z}$ is wrong without the condition $K \in \mathcal{W}_{\star}^{m, \nu ; \lambda, \mu}$.

Lemma 5.2. Assume that $K \in \mathcal{W}^{m, \nu ; \lambda, \mu}((a, b) \times(a, b)), m \geq k^{\prime}+1, \nu<1$, $\lambda, \mu<\min \{1,1-\nu\}$. Then the operators $T_{j}, j=1, \ldots, m-k^{\prime}$, and $T_{j}^{\star}$, $j=0, \ldots, m-k^{\prime}$, are bounded in $B C(a, b)$. For $\nu \notin \mathbb{Z}$, also $T_{0}$ is bounded in $B C(a, b)$; for $\nu \in \mathbb{Z}$ this is true if $m+\nu+\lambda-1 \leq 0, m+\nu+\mu-1 \leq 0$.

Proof. Denote

$$
R_{j}(x, y)=\tau_{j}(x, y)\left(\frac{\partial}{\partial x}\right)^{k^{\prime}+j} K(x, y), \quad j=0, \ldots, m-k^{\prime}
$$

where $\tau_{j}$ is defined by (4.7). Thus (see (5.2))

$$
\left(T_{j} u\right)(x)=w_{m+\nu+\lambda-1}(x-a) w_{m+\nu+\mu-1}(b-x) \int_{a}^{b} R_{j}(x, y) u(y) d y .
$$

Similarly as in the proof of Lemma 5.1 we represent $R_{j}(x, y)=R_{j}^{-}(x, y)+R_{j}^{+}(x, y)$ where $R_{j}^{-}(x, y)=R_{j}(x, y) \sigma(y)$ and $R_{j}^{+}(x, y)=R_{j}(x, y)(1-\sigma(y))$. We show that 
for $j \geq 0$ if $\nu \notin \mathbb{Z}$ and for $j \geq 1$ if $\nu \in \mathbb{Z}$, the following inequalities hold for $a<x<b$ :

$$
\begin{aligned}
& w_{m+\nu+\lambda-1}(x-a) \int_{a}^{b}\left|R_{j}^{-}(x, y)\right| d y \leq c \\
& w_{m+\nu+\mu-1}(b-x) \int_{a}^{b}\left|R_{j}^{+}(x, y)\right| d y \leq c .
\end{aligned}
$$

Clearly, (5.7) implies that $T_{j}$ is bounded in $B C(a, b)$ as asserted in the lemma. Due to symmetry, it suffices to establish the first one of inequalities (5.7).

Let us estimate $\left|R_{j}^{-}(x, y)\right|$. For $\tau_{j}(x, y)$ defined in (4.7) we have

$$
\left|\tau_{j}(x, y)\right| \leq c_{j}\left(\frac{\rho(x)}{2}\right)^{-\left(m-k^{\prime}-j\right)}, \quad c_{j}=\max _{r \geq 0}\left|\tau^{\left(m-k^{\prime}-j\right)}(r)\right| .
$$

Moreover, for $j \leq m-k^{\prime}$,

$$
\operatorname{supp} \tau_{j} \subset\left\{(x, y) \in[a, b] \times[a, b]:|x-y| \geq \frac{\rho(x)}{4}\right\}
$$

in particular, for $j<m-k^{\prime}$

$$
\operatorname{supp} \tau_{j} \subset\left\{(x, y) \in[a, b] \times[a, b]: \frac{\rho(x)}{4} \leq|x-y| \leq \frac{\rho(x)}{2}\right\}
$$

allowing to rewrite (5.8) in the form

$$
\left|\tau_{j}(x, y)\right| \leq c|x-y|^{-\left(m-k^{\prime}-j\right)}, \quad j=0, \ldots, m-k^{\prime}
$$

(for $j=m-k^{\prime}$ this estimate holds since $\tau_{m-k^{\prime}}(x, y)=\tau\left(2 \frac{|x-y|}{\rho(x)}\right)$.

Since $k^{\prime}+\nu=0$ for $\nu \in \mathbb{Z}$ and $0<k^{\prime}+\nu<1$ for $\nu \notin \mathbb{Z}$, estimate (1.3) yields

$$
\left|\left(\frac{\partial}{\partial x}\right)^{k^{\prime}+j} K(x, y)\right| \leq c|x-y|^{-\nu-k^{\prime}-j}(y-a)^{-\lambda}(b-y)^{-\mu}
$$

for $j \geq 0$ if $\nu \notin \mathbb{Z}$ and for $j \geq 1$ if $\nu \in \mathbb{Z}$. Composing the last two estimates we obtain $\left|R_{j}^{-}(x, y)\right| \leq c|x-y|^{-\nu-m}(y-a)^{-\lambda} \sigma(y)$; we took into account that $\sigma(y)$ cuts the singularity $(b-y)^{-\mu}$ off. Now

$$
\begin{aligned}
\int_{a}^{b}\left|R_{j}^{-}(x, y)\right| d y & \leq c \int_{(a, d) \backslash\{y:|x-y| \leq \rho(x) / 4\}}|x-y|^{-\nu-m}(y-a)^{-\lambda} d y \\
& \leq c \int_{(a, d) \backslash\{y:|x-y| \leq(x-a) / 8\}}|x-y|^{-\nu-m}(y-a)^{-\lambda} d y,
\end{aligned}
$$


where $d=a+\frac{2}{3}(b-a)$. With the change of variables $y-a=(x-a) z$ we find

$$
\begin{aligned}
& \int_{(a, d) \backslash\{y:|x-y| \leq(x-a) / 8\}}|x-y|^{-\nu-m}(y-a)^{-\lambda} d y \\
& =(x-a)^{-m-\nu-\lambda+1} \int_{(0,(d-a) /(x-a)) \backslash\left\{z:|1-z| \leq \frac{1}{8}\right\}}|1-z|^{-\nu-m} z^{-\lambda} d z \\
& \leq c(x-a)^{-m-\nu-\lambda+1}\left(1+\int_{\frac{9}{8}}^{\frac{d-a}{x-a}} z^{-m-\nu-\lambda} d z\right) \\
& \leq c^{\prime}(x-a)^{-m-\nu-\lambda+1} \begin{cases}(x-a)^{m+\nu+\lambda-1}, & m+\nu+\lambda-1<0 \\
1+|\log (x-a)|, & m+\nu+\lambda-1=0 \\
1, & m+\nu+\lambda-1>0\end{cases} \\
& =c^{\prime} \begin{cases}1, & m+\nu+\lambda-1<0 \\
1+|\log (x-a)|, & m+\nu+\lambda-1=0 \\
(x-a)^{-m-\nu-\lambda+1}, & m+\nu+\lambda-1>0\end{cases} \\
& =\frac{c^{\prime}}{w_{m+\nu+\lambda-1}(x-a)} \text {. }
\end{aligned}
$$

We obtained the first one of inequalities (5.7).

In the case $\nu \in \mathbb{Z}, j=0$, estimate (1.3) yields

$$
\left|\left(\frac{\partial}{\partial x}\right)^{k^{\prime}} K(x, y)\right| \leq c(y-a)^{-\lambda}(b-y)^{-\mu}(1+\log |x-y|) .
$$

Using (5.8) and (5.9) we may estimate directly

$$
\begin{aligned}
& \int_{a}^{b}\left|R_{0}^{-}(x, y)\right| d y \leq c \rho(x)^{-m-\nu-\lambda+1}(1+|\log \rho(x)|), \\
& \int_{a}^{b}\left|R_{0}^{+}(x, y)\right| d y \leq c \rho(x)^{-m-\nu-\mu+1}(1+|\log \rho(x)|) .
\end{aligned}
$$

For $m+\nu+\lambda-1 \leq 0, m+\nu+\mu-1 \leq 0$ we still have (5.7), and $T_{0}$ is bounded in $B C(a, b)$ (but it is not so if $m+\nu+\lambda-1>0$ or $m+\nu+\mu-1>0$ ).

Now consider the operators

$$
\left(T_{j}^{\star} u\right)(x)=w_{m+\nu+\lambda-1}^{\star}(x-a) w_{m+\nu+\mu-1}^{\star}(b-x) \int_{a}^{b} R_{j}(x, y) u(y) d y .
$$

Since $w_{s}^{\star}(x) \leq w_{s}(x)$, we have $\left|\left(T_{j}^{\star} u\right)(x)\right| \leq\left|\left(T_{j} u\right)(x)\right|$, and $T_{j}^{\star}$ is bounded in $B C(a, b)$ in all cases where $T_{j}$ is, in particular for $j \geq 1$. For $j=0,(5.10)$ implies

$$
w_{m+\nu+\lambda-1}^{\star}(x-a) \int_{a}^{b}\left|R_{0}^{-}(x, y)\right| d y \leq c, \quad a<x<b,
$$


hence also $T_{0}^{-, \star}, T_{0}^{+, \star}$ and $T_{0}^{\star}=T_{0}^{-, \star}+T_{0}^{+, \star}$ are bounded in $B C(a, b)$.

Lemma 5.3. Assume that $K \in \mathcal{W}_{\star}^{m, \nu ; \lambda, \mu}((a, b) \times(a, b)), m \geq k^{\prime}+1,1>\nu \in \mathbb{Z}$, $\lambda, \mu<1$. Then $T_{0}: C^{m, \nu+\lambda, \nu+\mu}(a, b) \rightarrow B C(a, b)$ is compact.

Proof. Let $u \in C^{m, \nu+\lambda, \nu+\mu}(a, b) \subset C[a, b]$. With the designations from the proof of Lemma 5.2, we represent

$$
\int_{a}^{b} R_{0}^{-}(x, y) u(y) d y=\int_{a}^{b} R_{0}^{-}(x, y)[u(y)-u(a)] d y+u(a) \int_{a}^{b} R_{0}^{-}(x, y) d y .
$$

To prove the Lemma, we show that

(i) the operator $R^{-}$defined by

$$
\left(R^{-} u\right)(x)=w_{m+\nu+\lambda-1}(x-a) \int_{a}^{b} R_{0}^{-}(x, y)[u(y)-u(a)] d y
$$

maps $C^{m, \nu+\lambda, \nu+\mu}(a, b)$ into $B C(a, b)$ and is compact between these spaces;

(ii) $\varphi^{-} \in B C(a, b)$ where the function $\varphi^{-}$is defined by

$$
\varphi^{-}(x)=w_{m+\nu+\lambda-1}(x-a) \int_{a}^{b} R_{0}^{-}(x, y) d y, \quad a<x<b .
$$

Similar claims for $R^{+}$and $\varphi^{+}$follow by the symmetry argument.

To claim (i). Fix an $\varepsilon>0$ such that $\lambda+\varepsilon<1$ and introduce the Banach space $C_{\varepsilon}^{-}[a, b]$ of continuous functions $u$ on $[a, b]$ with the finite norm

$$
\|u\|_{C_{\varepsilon}^{-}[a, b]}=\max _{a \leq x \leq b}|u(x)|+\sup _{a<x \leq b}(x-a)^{-\varepsilon}|u(x)-u(a)| .
$$

We have a compact imbedding $C^{m, \nu+\lambda, \nu+\mu}(a, b) \subset C_{\varepsilon}^{-}[a, b]$. Hence claim (i) follows noticing that $R^{-}: C_{\varepsilon}^{-}[a, b] \rightarrow B C(a, b)$ is bounded: from $|u(y)-u(a)| \leq$ $(y-a)^{\varepsilon}\|u\|_{C_{\varepsilon}^{-}[a, b]}$ we win the factor $\rho(x)^{\varepsilon}$ in the estimate (cf. (5.10))

$$
\left|\int_{a}^{b} R_{0}^{-}(x, y)[u(y)-u(a)] d y\right| \leq c \rho(x)^{-m-\nu-\lambda+1+\varepsilon}(1+|\log \rho(x)|)\|u\|_{C_{\varepsilon}^{-}[a, b]}
$$

implying

$$
\sup _{a<x<b} w_{m+\nu+\lambda-1}(x-a)\left|\int_{a}^{b} R_{0}^{-}(x, y)[u(y)-u(a)] d y\right| \leq c\|u\|_{C_{\varepsilon}^{-}[a, b]} .
$$

To claim (ii). If $m+\nu+\lambda-1 \leq 0$, (ii) is clear, since due to (5.10),

$$
\left|\varphi^{-}(x)\right| \leq w_{m+\nu+\lambda-1}(x-a) \int_{a}^{b}\left|R_{0}^{-}(x, y)\right| d y \leq c, \quad a<x<b .
$$


For $m+\nu+\lambda-1>0,(5.10)$ leads to an estimate $\left|\varphi^{-}(x)\right| \leq c(1+|\log (x-a)|)$ that is too coarse. So we have to deduce a finer estimate in the vicinity of the left boundary point $a$ in the case $m+\nu+\lambda-1>0$. Recall that

$$
\begin{aligned}
R_{0}^{-}(x, y) & =\tau_{0}(x, y)\left(\frac{\partial}{\partial x}\right)^{k^{\prime}} K(x, y) \sigma(y) \quad\left(k^{\prime}=-\nu\right) \\
\tau_{0}(x, y) & =\left[\left(\frac{\partial}{\partial x}\right)^{m-k^{\prime}} \tau\left(\frac{|x-y|}{r}\right)\right]_{r=\frac{\rho(x)}{2}} \\
& =(-1)^{m-k^{\prime}}\left(\frac{\partial}{\partial y}\right)^{m-k^{\prime}} \tau\left(\frac{2|x-y|}{\rho(x)}\right)
\end{aligned}
$$

Integrating $m-k^{\prime}$ times by part we represent

$$
\begin{aligned}
\int_{a}^{b} R_{0}^{-} & (x, y) d y \\
& =(-1)^{m-k^{\prime}} \int_{x-\frac{\rho(x)}{2}}^{x+\frac{\rho(x)}{2}}\left(\frac{\partial}{\partial x}\right)^{k^{\prime}} K(x, y) \sigma(y)\left(\frac{\partial}{\partial y}\right)^{m-k^{\prime}} \tau\left(\frac{2|x-y|}{\rho(x)}\right) d y \\
& =\int_{x-\frac{\rho(x)}{2}}^{x+\frac{\rho(x)}{2}}\left\{\left(\frac{\partial}{\partial x}\right)^{k^{\prime}}\left(\frac{\partial}{\partial y}\right)^{m-k^{\prime}} K(x, y) \sigma(y)\right\} \tau\left(\frac{2|x-y|}{\rho(x)}\right) d y+\beta^{-}(x)
\end{aligned}
$$

where

$$
\beta^{-}(x)=-\left[\left(\frac{\partial}{\partial x}\right)^{k^{\prime}}\left(\frac{\partial}{\partial y}\right)^{m-k^{\prime}-1} K(x, y) \sigma(y)\right]_{y=x-\frac{\rho(x)}{2}}^{y=x+\frac{\rho(x)}{2}}
$$

we assume here that the cutting function $\sigma$ (see (2.2)) is chosen from $C^{m}[a, b]$. Since $\sigma(y)=1$ for $a \leq y \leq a+\frac{1}{3}(b-a)$, we have for $a<x \leq x_{0}=a+\frac{2}{9}(b-a)$,

$\int_{a}^{b} R_{0}^{-}(x, y) d y=\int_{x-\frac{x-a}{2}}^{x+\frac{x-a}{2}}\left\{\left(\frac{\partial}{\partial x}\right)^{k^{\prime}}\left(\frac{\partial}{\partial y}\right)^{m-k^{\prime}} K(x, y)\right\} \tau\left(\frac{2|x-y|}{x-a}\right) d y+\beta^{-}(x)$

where

$$
\beta^{-}(x)=-\left[\left(\frac{\partial}{\partial x}\right)^{k^{\prime}}\left(\frac{\partial}{\partial y}\right)^{m-k^{\prime}-1} K(x, y)\right]_{y=x-\frac{x-a}{2}}^{y=x+\frac{x-a}{2}} .
$$

Further, we expand

$$
\begin{aligned}
\left(\frac{\partial}{\partial y}\right)^{m-k^{\prime}} & =\left(\left(\frac{\partial}{\partial x}+\frac{\partial}{\partial y}\right)-\frac{\partial}{\partial x}\right)^{m-k^{\prime}} \\
& =\sum_{j=0}^{m-k^{\prime}}(-1)^{j}\left(\begin{array}{c}
m-k^{\prime} \\
j
\end{array}\right)\left(\frac{\partial}{\partial x}+\frac{\partial}{\partial y}\right)^{m-k^{\prime}-j}\left(\frac{\partial}{\partial x}\right)^{j}
\end{aligned}
$$


and similarly $\left(\frac{\partial}{\partial y}\right)^{m-k^{\prime}-1}$ in $\beta^{-}(x)$. For $j \geq 1$ we estimate on the basis of (1.3)

$$
\left|\left(\frac{\partial}{\partial x}\right)^{k^{\prime}+j}\left(\frac{\partial}{\partial x}+\frac{\partial}{\partial y}\right)^{m-k^{\prime}-j} K(x, y)\right| \leq c|x-y|^{-j}(x-a)^{-m-\nu-\lambda+j}
$$

and for $j=0$ on the basis of (1.4)

$$
\left|\left(\frac{\partial}{\partial x}\right)^{k^{\prime}}\left(\frac{\partial}{\partial x}+\frac{\partial}{\partial y}\right)^{m-k^{\prime}} K(x, y)\right| \leq c(1+|\log | x-y||) \frac{(x-a)^{-\lambda-m-\nu}}{1+|\log (x-a)|}
$$

note that $\lambda+\left(m-k^{\prime}\right)=m+\nu+\lambda>0$, even $\lambda+\left(m-k^{\prime}\right)>1$, so the use of (1.4) is legitime, and it remains to be legitime also when we estimate the corresponding term of $\beta^{-}(x)$. Observe that $\tau\left(\frac{2|x-y|}{x-a}\right)=0$ for $|x-y| \leq \frac{x-a}{4}$, thus the integration interval $\left(x-\frac{x-a}{2}, x+\frac{x-a}{2}\right)$ actually reduces to the union of intervals $\left(x-\frac{x-a}{2}, x-\frac{x-a}{4}\right)$ and $\left(x+\frac{x-a}{4}, x+\frac{x-a}{2}\right)$ in which the quantities $|x-y|$ and $x-a$ are of the same order. So the estimates reduce to

$$
\left|\left(\frac{\partial}{\partial x}\right)^{k^{\prime}+j}\left(\frac{\partial}{\partial x}+\frac{\partial}{\partial y}\right)^{m-k^{\prime}-j} K(x, y)\right| \leq c(x-a)^{-m-\nu-\lambda}
$$

for $j \geq 1$ as well as for $j=0$, and

$$
\left|\int_{a}^{b} R_{0}^{-}(x, y) d y\right| \leq c(x-a)^{-m-\nu-\lambda+1}, \quad a<x \leq x_{0} .
$$

Recalling that $m+\nu+\lambda-1>0$, this implies

$$
\left|\varphi^{-}(x)\right| \leq w_{m+\nu+\lambda-1}(x-a)\left|\int_{a}^{b} R_{0}^{-}(x, y) d y\right| \leq c, \quad a<x \leq x_{0},
$$

as desired.

Finally, we turn to the case $1 \leq m \leq k^{\prime}$; then $\nu<0, \nu+m<1$.

Lemma 5.4. Assume that $K \in \mathcal{W}^{m, \nu ; \lambda, \mu}((a, b) \times(a, b)), 1 \leq m \leq k^{\prime}, \lambda<1$, $\mu<1$. Then the integral operator $T_{m}^{\prime}$ defined by (cf. (4.8))

$$
\left(T_{m}^{\prime} u\right)(x)=w_{m+\nu+\lambda-1, m+\nu+\mu-1}(x) \int_{a}^{b}\left(\frac{\partial}{\partial x}\right)^{m} K(x, y) u(y) d y, \quad a<x<b,
$$

is bounded in the space $B C(a, b)$. 
Proof. Inequality (1.3) yields

$$
\left|\left(\frac{\partial}{\partial x}\right)^{m} K(x, y)\right| \leq c \kappa_{\nu+m}(|x-y|)(y-a)^{-\lambda}(b-y)^{-\mu},
$$

and in accordance to Remark 2.3 we have

$$
\begin{aligned}
& \int_{a}^{b}\left|\left(\frac{\partial}{\partial x}\right)^{m} K(x, y)\right| d y \leq c\left\{\begin{array}{ll}
1, & \nu+m+\lambda<1 \\
1+|\log (x-a)|, & \nu+m+\lambda=1 \\
(x-a)^{1-\nu-m-\lambda}, & \nu+m+\lambda>1
\end{array}\right\} \\
&+c\left\{\begin{array}{ll}
1, & \nu+m+\mu<1 \\
1+|\log (b-x)|, & \nu+m+\mu=1 \\
(b-x)^{1-\nu-m-\mu}, & \nu+m+\mu>1
\end{array}\right\}
\end{aligned}
$$

Hence

$$
w_{m+\nu+\lambda-1, m+\nu+\mu-1}(x) \int_{a}^{b}\left|\left(\frac{\partial}{\partial x}\right)^{m} K(x, y)\right| d y \leq c, \quad a<x<b,
$$

that proves the assertion of the Lemma.

We are ready to complete the proof of Lemmas 1.8 and 1.9. Actually we already have constructed all the details we need and we only tell how to compose them.

Proof of Lemma 1.8. Let $K \in \mathcal{W}^{m, \nu ; \lambda, \mu}((a, b) \times(a, b))$ where $m \geq 1, \nu<1$, $\lambda, \mu<\min \{1,1-\nu\}$. Recall the definition (4.5) of $k^{\prime}$.

In the case $1 \leq m \leq k^{\prime}$, the compactness of $T_{K}$ in $C^{m, \nu+\lambda, \nu+\mu}(a, b)$ immediately follows from (4.8) on the basis of Lemma 1.7, Lemma 3.4, Lemma 5.4 and the compactness of the imbeddings (1.7).

In the case $m>k^{\prime}$ we use formulae (5.1)-(5.3), Lemmas 5.1-5.2 and still Lemma 1.7, Lemma 3.4 and the compactness of the imbeddings (1.7).

Proof of Lemma 1.9. The proof of Lemma 1.9 is composed in a similar way as the proof of Lemma 1.8 adding Lemma 5.3 to the list of details in the case $m>k^{\prime}$.

Together with the proof of Lemmas 1.8 and 1.9, also the proof of the Theorems 1.2 and 1.3 is complete.

Acknowledgement.. This work was supported by Estonian Science Foundation, Grant 5859. The authors express their gratitude to Raul Kangro for a very valuable critical remark. 


\section{References}

[1] Atkinson, K. E., The Numerical Solution of Integral Equations of the Second Kind. Cambridge: Cambridge Univ. Press 1997.

[2] Brunner, H., Nonpolynomial spline collocation for Volterra equations with weakly singular kernels. SIAM J. Numer. Anal. 20 (1983), 1106 - 1119.

[3] Brunner, H., Collocation Methods for Volterra Integral and Related Functional Equations. Cambridge: Cambridge Univ. Press 2004.

[4] Brunner, H. and P.J. van der Houwen,P. J., The Numerical Solution of Volterra Equations. Amsterdam: North-Holland, 1986.

[5] Brunner, H., A. Pedas, A. and Vainikko, G., The piecewise polynomial collocation method for nonlinear weakly singular Volterra equations. Math. Comp. 68 (1999), 1079 - 1095.

[6] Cao, Y. and Xu, Y., Singularity preserving Galerkin methods for weakly singular Fredholm integral equations. J. Integral Equations Appl. 6 (1994), $303-334$.

[7] Graham, I. G., Singularity expansions for solutions of second kind Fredholm integral equations with weakly singular convolution kernels. J. Integral Equations Appl. 4 (1982), 1 - 30.

[8] Kaneko, H., Noren, R. D. and Padilla, P. A., Singularity preserving Galerkin method for Hammerstein equations with logarithmic kernel. Adv. Comput. Math. 9 (1998), 363 - 376.

[9] Kaneko, H., Noren, R. D. and Xu, Y., Regularity of the solution of Hammerstein equations with weakly singular kernel. Integral Equations Operator Theory 13 (1990), 660 - 670.

[10] Kangro, R., On the smoothness of solutions to an integral equation with a kernel having a singularity on a curve. Acta et Comm. Univ. Tartuensis 913 (1990), $24-37$.

[11] Kangro, U., The smoothness of the solution to a two-dimensional integral equation with logarithmic kernel. Z. Anal. Anwendungen 12 (1993), 305 - 318.

[12] Lions, J. L. and Magenes, E., Non-Homogeneous Boundary Value Problems and Applications, Vol. 1. Berlin: Springer 1972.

[13] Miller, R. K. and Feldstein, A., Smoothness of solutions of Volterra integral equations with weakly singular kernels. SIAM J. Math. Anal. 2 (1971), $242-258$.

[14] Pedas, A., On the smoothness of the solution of integral equation with a weakly singular kernel (in Russian). Acta et Comm. Univ. Tartuensis 492 (1979), $56-68$.

[15] Pedas, A. and Vainikko, G., The smoothness of solutions to nonlinear weakly singular integral equations. Z. Anal. Anwendungen 13 (1994), 463 - 476.

[16] Pedas, A. and Vainikko, G., Boundary singularities of solutions to integral equations of the second kind. WSEAS Transactions Math. 4 (2005), $70-75$. 
[17] Pitkäranta, J., Estimates for the derivatives of solutions to weakly singular Fredholm integral equations. SIAM J. Math. Anal. 11 (1980), 952 - 968.

[18] Richter, G. R., On weakly singular Fredholm integral equations with displacement kernels. J. Math. Anal. Appl. 55 (1976), 35 - 42.

[19] Schneider, C., Regularity of the solution to class of weakly singular Fredholm integral equations of the second kind. Integral Equations Operator Theory 2 (1979), $62-68$.

[20] Uba, P., Smoothness of the solution of a weakly singular integral equation with a discontinuous coefficient (in Russian). Proc. Estonian Acad. Sci. Phys. Math. 37 (1988), $192-203$.

[21] Vainikko, G., On the smoothness of the solution of multidimensional weakly singular integral equations (in Russian). Mat. Sb. 180 (1989)(12), 1709 - 1723; translation in: Math. USSR-Sb. 68 (1991)(2), $585-600$.

[22] Vainikko, G., Multidimensional Weakly Singular Integral Equations. Lecture Notes Math. 1549. Berlin: Springer 1993.

[23] Vainikko, G. and Pedas,A., The properties of solutions of weakly singular integral equations. J. Austral. Math. Soc. Ser. B 22 (1980/81)(4), $419-430$.

[24] Vainikko, G., Pedas, A. and Uba, P., Methods for Solving Weakly Singular Integral Equations (in Russian). Tartu: Tartu. Gos. Univ. 1984.

Received April 6, 2005; revised October 7, 2005 